\title{
YAP induces high-grade serous carcinoma in fallopian tube secretory epithelial cells
}

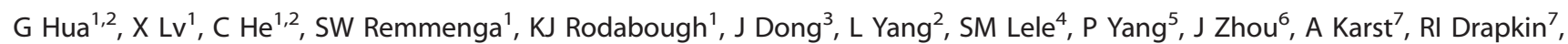 \\ JS Davis ${ }^{1,3,8}$ and C Wang ${ }^{1,3}$
}

Accumulating evidence indicates that ovarian high-grade serous carcinoma (HGSC) originates from fallopian tube secretory epithelial cells (FTSECS). However, the molecular mechanisms underlying the initiation and progression of HGSC derived from FTSECs remains unclear. In this study, we found that the Hippo/Yes-associated protein (YAP) signaling pathway has a critical role in the initiation and progression of fallopian tube and ovarian HGSC. Importantly, YAP was overexpressed in inflammatory and cancerous fallopian tube tissues. Further, overexpression of wild-type YAP, or constitutively active YAP in immortalized FTSECs, induced cell proliferation, migration, colony formation and tumorigenesis. Moreover, the Hippo/YAP and the fibroblast growth factor (FGF) signaling pathways formed an autocrine/paracrine-positive feedback loop to drive the progression of the FTSECderived HGSC. Evidence in this study strongly suggests that combined therapy with inhibitors of YAP (such as verteporfin) and FGF receptors (such as BGJ398) can provide a novel therapeutic strategy to treat fallopian tube and ovarian HGSC.

Oncogene (2016) 35, 2247-2265; doi:10.1038/onc.2015.288; published online 14 September 2015

\section{INTRODUCTION}

Ovarian cancer is the most lethal gynecological cancer. Globally, approximately 225500 women are diagnosed with ovarian cancer annually, with an estimated 140200 associated deaths worldwide. ${ }^{1}$ The majority $(\sim 80 \%)$ of ovarian cancers are of epithelial origin. A key feature of high-grade serous carcinoma (HGSC), which constitutes $60-80 \%$ of ovarian epithelial carcinomas, is its aggressive nature and its unique genetic alterations. ${ }^{2,3}$ Patients with HGSC most frequently present at advanced clinical stages and have a very poor overall survival.

The etiology of ovarian HGSC is unclear. Previous studies suggest that HGSC is derived from the neoplastic transformation of ovarian surface epithelial cells in the cortical inclusion cysts of the ovary. ${ }^{4,5}$ However, the existence of a precursor lesion in the ovary that leads to HGSC has not been demonstrated conclusively. ${ }^{6,7}$ Studies using ovarian and fallopian tube specimens from prophylactic salpingo-oophorectomy of BRCA1/2mutation carriers suggest that most ovarian HGSC originate in the fimbrial end of the fallopian tube. ${ }^{8,9}$ Recent studies indicate that ovarian HGSC, primary peritoneal carcinoma and fallopian tube cancer (FTC) have similar pathogenesis and may originate from the same cell source, the fallopian tube secretory epithelial cells (FTSECs). ${ }^{10}$ Epidemiological studies also support the concept that ovarian, fallopian tube and primary peritoneal cancers have a common etiology. ${ }^{11}$ Obviously, the conventional pathologic classification of many other pelvic serous cancers primarily as ovarian cancer contributes to underreporting the incidence of the FTC because, in many cases, FTCs are also present on the surface of the ovary. The involvement of ovary in conventional ovarian
HGSC is potentially a secondary event. Therefore, studies on the mechanisms underlying the initiation and progression of fallopian tube HGSC represent a new and promising direction for the diagnosis and treatment of ovarian cancer.

The etiology of FTC is also unknown. Recent studies suggest that disruption of the Hippo pathway is an important oncogenic event during tumorigenesis in many cancers. ${ }^{12,13}$ First discovered in Drosophila, ${ }^{14,15}$ the Hippo pathway is a growth control pathway that is highly conserved throughout species. ${ }^{16}$ Accumulating evidence indicates that the Hippo pathway has a fundamental role in organ size control, stem cell function and tumor suppression. Hence, the Hippo pathway has attracted growing interest. ${ }^{12,13,16}$ Activation of the Hippo pathway suppresses the activity of the transcriptional co-activator Yes-associated protein 1 (YAP1, commonly referred to as YAP) by phosphorylating YAP and subsequently retaining it in the cytoplasm. YAP has been identified as an ovarian cancer oncogene. ${ }^{17,18}$ Our research also indicates that YAP contributes to ovarian cancer progression. ${ }^{19,20}$ Although several very recent studies indicate the importance of FTSECs in the tumorigenesis of the fallopian and ovarian HGSC, $^{11-14,21,22}$ the extent to which the Hippo pathway is involved in their initiation and progression has not been examined.

In addition to limited information on the etiology, the molecular mechanism underlying the rapid progression of fallopian tube and ovarian HGSCs is also unclear. Interestingly, previous studies have shown that cultured human FTSECs produce basic fibroblast growth factor 2 (FGF2). ${ }^{23}$ FGF2, a growth regulatory peptide secreted from cells, is reported to be involved in a variety of biological processes including cell differentiation, cell growth,

\footnotetext{
${ }^{1}$ Olson Center for Women's Health, Department of Obstetrics/Gynecology, University of Nebraska Medical Center, Omaha, NE, USA; ${ }^{2}$ The Key Lab of Agricultural Animal

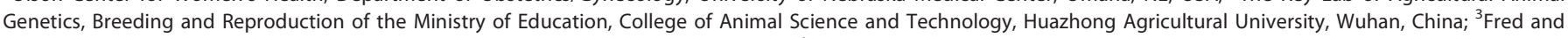
Pamela Buffett Cancer Center, University of Nebraska Medical Center, Omaha, NE, USA; ${ }^{4}$ Department of Pathology and Microbiology, University of Nebraska Medical Center, Omaha, NE, USA; ${ }^{5}$ Department of Obstetrics, Gynecology and Reproductive Sciences, University of Maryland School of Medicine, Baltimore, MD, USA; ${ }^{6}$ Department of Obstetrics and Gynecology, Urumuqi General Hospital of Lanzhou Military Region, Urumuqi, China; ${ }^{7}$ Dana-Farber Cancer Institute, Harvard Medical School, Boston, MA, USA and ${ }^{8}$ Omaha Veterans Affairs Medical Center, Omaha, NE, USA. Correspondence: Dr C Wang, Olson Center for Women's Health, Department of Obstetrics/Gynecology, University of Nebraska Medical Center, Fred and Pamela Buffett Cancer Center, 985860 Nebraska Medical Center, Omaha, NE 68198-6265, USA.
} 
migration, angiogenesis and tumor formation. ${ }^{24}$ Most importantly, several phase I and phase II clinical trials for a pan FGF receptor (FGFR) inhibitor, BGJ398, ${ }^{25}$ are currently underway to examine the role of this novel small molecule in the treatment of several solid tumors (http://clinicaltrials.gov/ ct2/results?term = BGJ398\&Search= Search). However, it is not known whether FGF2 secreted by FTSECs contribute to the tumorigenic process of fallopian tube and ovarian HGSC. Whether the Hippo/YAP signaling pathway interacts with the FGF/FGFR signaling pathway to regulate the rapid progression of fallopian tube and ovarian HGSC has not been investigated.

In turn, this study aims to determine if the Hippo/YAP signaling pathway is involved in initiation and progression of fallopian tubederived HGSC and the potential signaling mechanism(s) underlying the Hippo/YAP pathway regulation of HGSC initiation and progression.

\section{RESULTS}

Expression of YAP in normal and cancerous human fallopian tube tissues

Immunohistochemical analysis of normal and cancerous fallopian tube tissues showed that the YAP immunosignal in normal fallopian tube tissues was very low (Figures $1 a$ and $b$, Supplementary Figure 1). In the normal fallopian tube tissues, the YAP immunosignal was localized to both cytoplasm and nucleus of some epithelial cells (Supplementary Figure 1). However, the YAP immunosignal was significantly increased in inflammatory (chronic tube inflammation) and cancerous tissues (Figures 1a and b, Supplementary Figure 1). Also, the immunosignal was mainly localized to nuclei of almost all epithelial cells of these abnormal tissues (Supplementary Figure 1). The immunosignal positivity and intensity in the cancerous tissues significantly increased compared with that of normal and inflammatory tissues (Figures $1 \mathrm{a}$ and $\mathrm{b} ; P<0.001$ ). Further, the YAP immunosignal positivity and intensity in the inflammatory tissues were also significantly increased compared with normal tissues (Figures 1a and $\mathrm{b} ; P<0.05)$.

Expression and alteration of YAP gene in ovarian HGSC

As most ovarian HGSCs are believed to originate from fallopian tube HGSC, we detected the expression of YAP protein in ovarian HGSC with a human tissue microarray. Our results also indicate that both the positivity (Figure 1c) and intensity (Figure 1d) of YAP immunosignal in the ovarian HGSCs $(n=105)$ are significantly higher than in the normal ovarian tissues $(n=42)(P<0.001)$. Moreover, the YAP immunosignal was present mainly in the nuclei of the ovarian HGSC cancer cells (Supplementary Figure 2). To further confirm the importance of YAP in the ovarian HGSC, we performed multidimensional genomic data analysis using online cancer databases and analysis tools (The Cancer Genomic Atlas (TCGA) and the cBioPortal for Cancer Genomics). ${ }^{26,27}$ Mining these databases revealed that the YAP gene is frequently amplified in ovarian HGSC (7.9\% amplification vs $0.7 \%$ deletion, $n=279$ ) (Figures $1 \mathrm{e}$ and $\mathrm{f}$, Supplementary Table 1). ${ }^{28,29}$ Cervical cancer has the highest YAP gene alteration across all gynecological cancers (Figure 1e), which is consistent with our recent finding (manuscript accepted for publication in EMBO Molecular Medicine). LATS1 is a core component of the Hippo/YAP signaling cascade and a major negative regulator of YAP activity, whereas TEAD is the major mediator of YAP activity. ${ }^{13,16}$ In examined ovarian HGSC patient samples, LATS1 gene is greatly downregulated, whereas YAP and TEADs are upregulated (Figure 1f), confirming our hypothesis that the Hippo/YAP pathway has critical roles in the progression of ovarian HGSC. In the ovarian HGSC samples, although YAP/TEAD expression and survival rates are not significantly correlated (total $(n=418)$ vs YAP/TEAD down $(n=19)$,
$P=0.1003)$, the result indicated a trend that downregulation of YAP gene was associated with a higher overall survival rate (Figure 1g).

Establishment and characterization of YAP-overexpressing FTSEC cell lines

The five non-tumorigenic FTSEC cell lines used in this study were characterized previously. ${ }^{30,31}$ As expected, western blot analysis showed that all FTSEC cell lines retained expression of Müllerian (PAX8) and epithelial (cytokeratin-7) lineage markers (Supplementary Figure 3a). Further, western blot results indicated that these cell lines have differential expression and phosphorylation of YAP protein (Supplementary Figure 3a). Both total and phosphorylated YAP levels in FT194, FT237 and FT240 cells were higher than that in FT190 and FT246 cells. As the TP53 protein was inactivated by SV40 large T antigen in FT190 and FT194 cells and was knocked down in FT237, FT240 and FT246 cells, ${ }^{30}$ it is difficult to estimate the association between YAP expression and TP53 protein levels. To examine the role of YAP in the proliferation of FTSECs, we established six cell lines with differential levels of expression and activation of YAP protein based on FT194 (with virus-inactivated TP53) and FT246 (with TP53 knockdown) cell lines. Fluorescent immunohistochemical analyses indicated that YAP expression levels in FT194-YAP, FT246-YAP, FT194-YAP ${ }^{5127 A}$ and FT246-YAP $127 \mathrm{~A}$ cells markedly increased compared with FT194-MXIV and FT246-MXIV control cells (Figure 2a). Importantly, YAP was primarily localized in the nuclei of FT194-YAP ${ }^{5127 A}$ and FT246-YAP ${ }^{\text {S127A }}$ cells (Figure 2a). Overexpression of YAP also induced a significant change in the morphology of FTSECs. YAP and YAP ${ }^{S 127 A}$-overexpressing cells are elongated and develop a spindle-shaped morphology (Figure 2a, Supplementary Figure 4). Consistent with the immunofluorescence results, western blot results showed that FT194-YAP and FT246-YAP cells, which were transfected with vectors expressing wild-type YAP, had significantly increased levels of YAP and phosphorylated YAP (Figure 2b, Supplementary Figures 3b and c). FT194-YAP $127 \mathrm{~A}$ and FT246-YAP ${ }^{\text {S127A }}$ cells, which were transfected with mutated YAP (constitutive activation mutation), however, have high levels of YAP protein and very low levels of phosphorylated YAP because of the mutation of YAP at serine 127.

YAP promotes growth and induces transformation of FTSEC cells in vitro

Consistent with the differential expression and cellular distributions of YAP protein in established stable cell lines, proliferation of these cells varied significantly. For example, FT194-MXIV and FT246-MXIV cells grew slowly, and their growth was inhibited when cells reach confluence (Figure $2 b$, Supplementary Figure $3 c$ ).

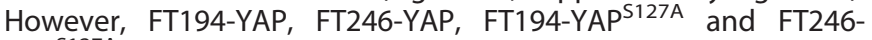
$Y A P^{5127 A}$ cells continued to grow after they reached confluence. Also, FT194-YAP ${ }^{\text {127A }}$ and FT246-YAP ${ }^{\text {S127A }}$ had significantly increased growth rates compared with corresponding wild-type YAP-transfected cells (FT194-YAP and FT246-YAP) (Figure 2b, Supplementary Figure $3 \mathrm{~b}$ ). In agreement with these observations, knockdown of YAP in FT194 and FT246 cells significantly suppressed their proliferation (Figures $2 \mathrm{c}$ and $\mathrm{d}$ ). The growthpromoting effect of YAP in FTSECs was also confirmed by the MTT assay (Supplementary Figures $5 \mathrm{a}$ and b). The pro-proliferation activity of YAP in FTSECs was further indicated by its action on the progression of cell cycle. Ectopic expression of wild-type YAP or constitutively active YAP in FT194 and FT246 cell lines promoted cell cycle progression, as indicated by significant increases in the proportion of cells in S and G2/M phase and decreases in cells in the G1 phase (Supplementary Figures $6 a$ and $c$ ). These results were further confirmed by increases in the cyclin A, cyclin B and

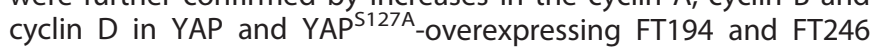
cell lines compared with their corresponding control cell lines 
a

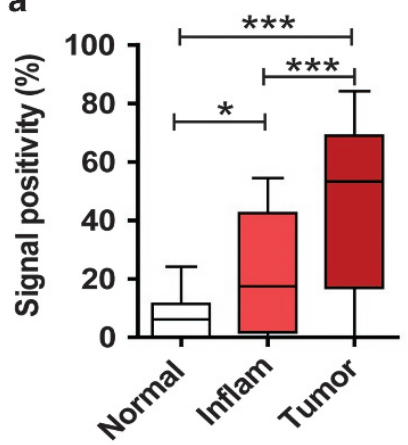

d

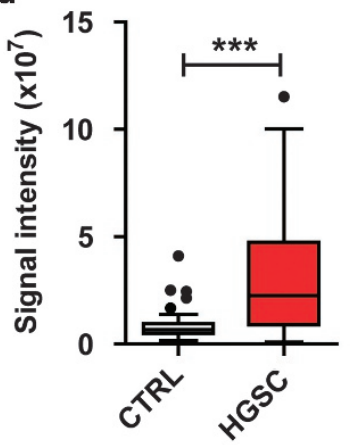

b

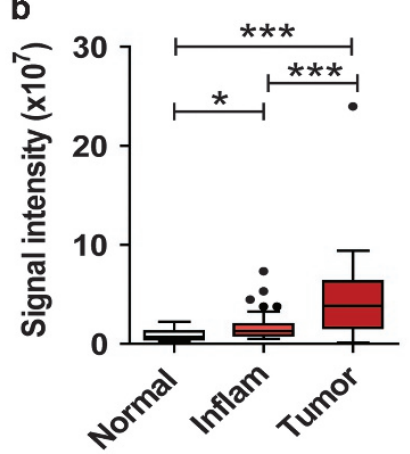

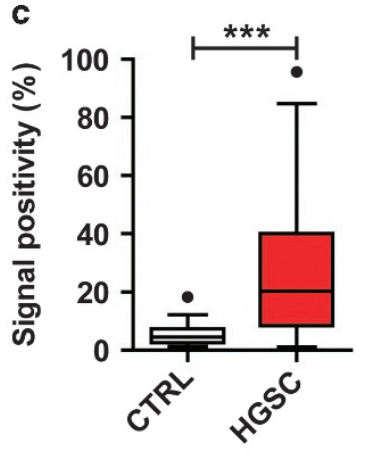

e

Gynecological cancers

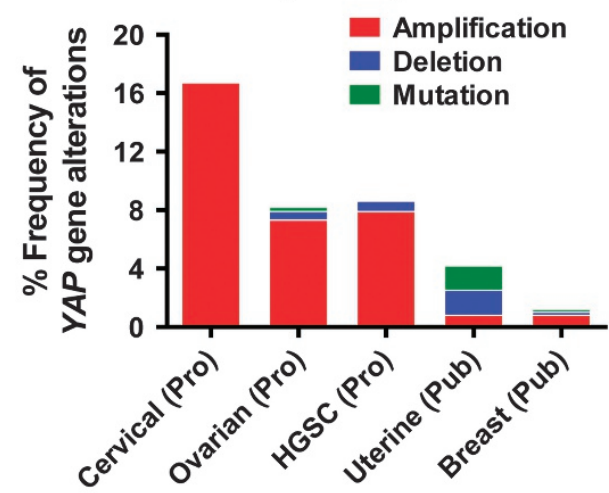

f

Ovarian HGSC
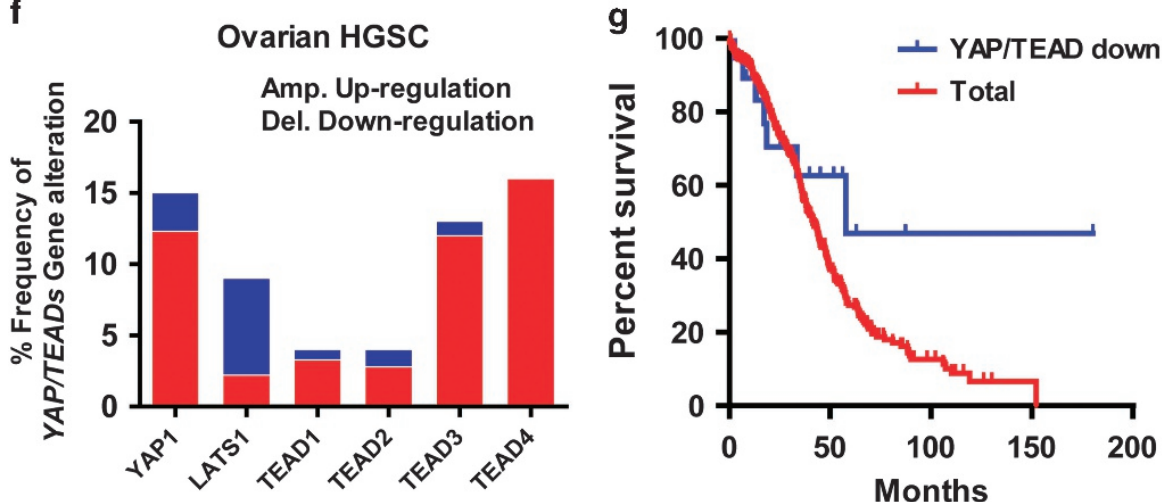

Figure 1. Expression of YAP protein and alterations of YAP-associated genes in FTC and ovarian HGSC. (a) Quantitative data showing YAP immunosignal positivity (percentage of the YAP-positive cell number relative to the total cell number) in normal, inflammatory (chronic tube inflammation, Inflam) and cancerous (tumor) fallopian tube tissues determined by immunohistochemistry. (b) Quantitative data showing YAP immunosignal intensity in normal, inflammatory (Inflam) and cancerous fallopian tube tissues determined by immunohistochemistry. Each bar represents the mean \pm s.e.m. ( $n=5$ for normal, $n=10$ for Inflam, $n=10$ for tumor. ${ }^{*} P<0.05$, ${ }^{* *} P<0.001$ ). (c) Quantitative data showing YAP immunosignal positivity in the ovarian normal tissues and ovarian HGSC. (d) Quantitative data showing YAP immunosignal intensity in the ovarian normal tissues and ovarian HGSC. CTRL, normal ovarian tissues used as control; HGSC, ovarian HGSC tissues. Each bar represents the mean \pm s.e.m. ( $n=42$ for CTRL, $n=105$ for HGSC). ${ }^{* *} P<0.001$. (e) Alterations of $Y A P$ gene across the gynecological cancers. The cross-cancer YAP gene alteration analyses were performed using online data sets and data-mining tools (the cBioPortal for Cancer Genomics and the data sets from the TCGA research Network). (f) Alterations of YAP, LATS1 and TEAD genes in the ovarian HGSC. Data sources and analysis tools are the same as in e. (g) Correlation between overall survival and YAP/TEAD expression in the ovarian HGSC. Data were extracted from the TCGA data sets using the cBioPortal and uploaded to the GraphPad Prism 5 for statistical analysis. Total: correlation between YAP/TEAD levels and overall survival in all ovarian HGSC cases $(n=418)$; YAP/TEAD down: correlation between YAP/TEAD expression levels and the overall survival in 19 cases of ovarian HGSC in which YAP/TEAD expression levels are lower and are out of the default Z-score thresholds.

(MXIV-transfected cells) (Supplementary Figures $6 \mathrm{~b}$ and $\mathrm{d}$ ). Consistent with these observations, small interfering RNA (siRNA) knockdown of YAP with YAP siRNA significantly reduced the proportion of cells in the $S$ and G2/M phase and increased the proportion of cells in G1 phase (Supplementary Figure 7).

It has been shown that the immortalized FTSECs, like primary fallopian tube cells, demonstrate regular contact inhibition during growth. ${ }^{30}$ The continued growth of FT194-YAP, FT246-YAP, FT194-
YAP ${ }^{\text {S127A }}$ and FT246-YAP ${ }^{\text {S127A }}$ cells after reaching confluence suggests that these cells are transformed following YAP overexpression. Soft agar assays showed that FT194-MXIV and FT246-MXIV cells did not form colonies in soft agar (Figures 3a and b). However, FT194-YAP, FT246-YAP, FT194-YAP ${ }^{5127 A}$ and FT246-YAP $127 A$ cells formed colonies in soft agar (Figure 3). FT194-YAP ${ }^{\text {S127A }}$ and FT246-YAP ${ }^{\text {127A }}$ cells formed a greater number of and larger colonies compared with FT194-YAP and FT246-YAP cells, 
a
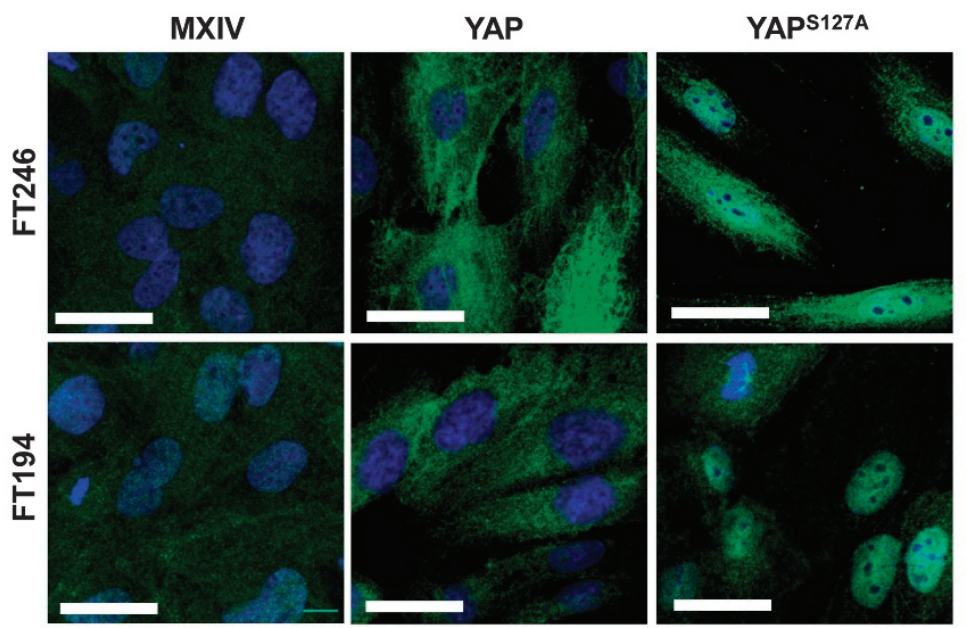

b
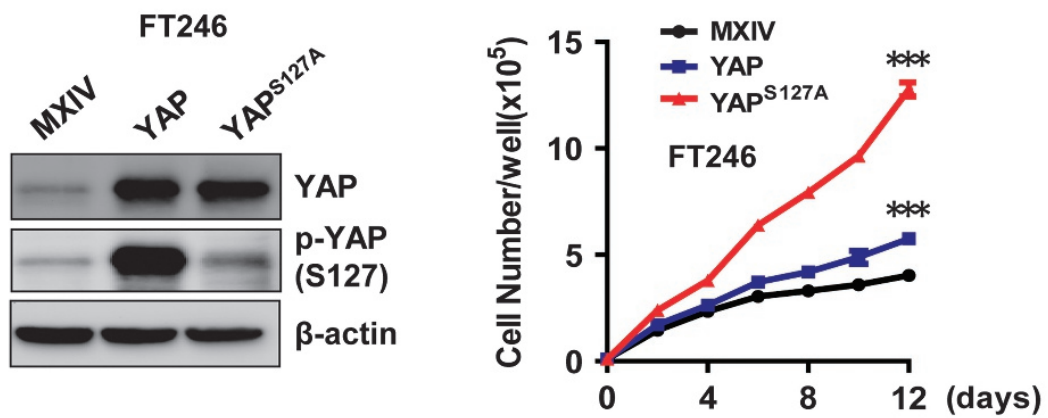

c
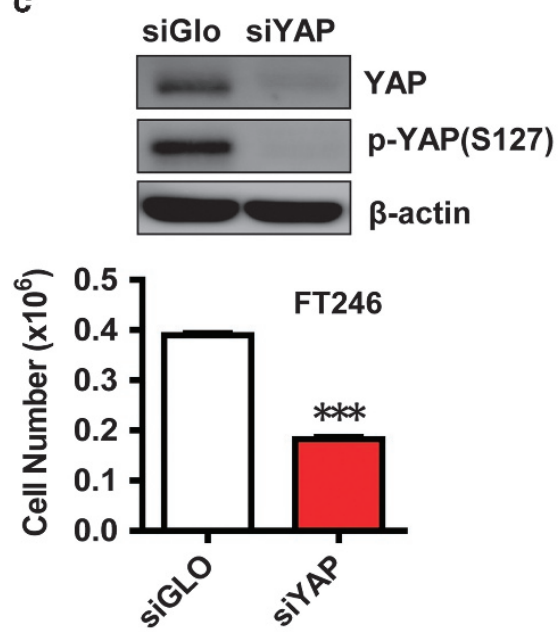

d
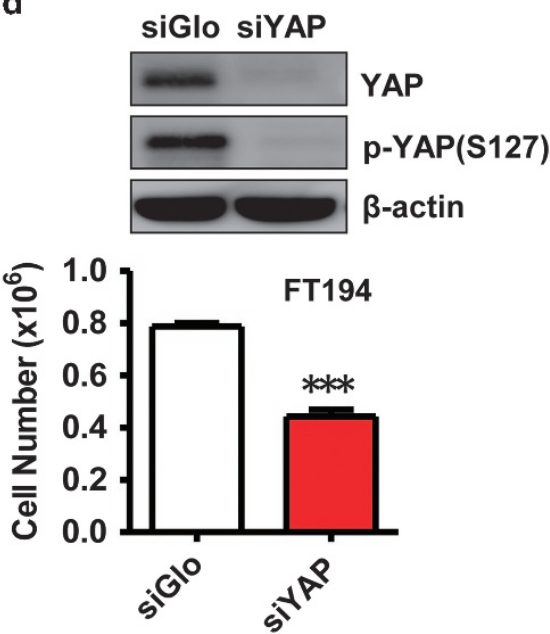

Figure 2. Effect of YAP protein levels on the proliferation of FTSECs. (a) Fluorescent immunocytochemistry determining the expression and localization of YAP protein (green) in FT246-MXIV, FT246-YAP and FT246-YAP ${ }^{\text {S127A }}$ and FT194-MXIV, FT194-YAP and FT194-YAP ${ }^{\text {S127A }}$ cell lines. Nuclei were stained with DAPI (blue). Scale bar $=50 \mu \mathrm{m}$. (b) Overexpression of wild-type YAP or constitutively active YAP on the proliferation of FT246 cells. Left panel: western blot detection of levels of YAP and phosphorylated YAP in FT246-MXIV, FT246-YAP and FT246-YAPS127A cell lines. Right panel: growth curves of FT246-MXIV, FT246-YAP and FT246-YAP ${ }^{S 127 A}$ cell lines. ${ }^{* * *} P<0.001$ compared with MXIV control. (c) Top: protein levels of YAP and phosphorylated YAP (ser127) in FT246 cells with or without knockdown of YAP with YAP siRNA (siYAP). SiGlo: nontargeting siRNA used as a control. Bottom: effect of YAP knockdown on the proliferation of FT246 cells. ${ }^{* * *} P<0.001$ compared with siGlo control. (d) Effect of knockdown of YAP on the proliferation of FT194 cells. ${ }^{* * *} P<0.001$ compared with siGlo control.

respectively (Figures $3 a$ and b). A fluorescence-based cell transformation assay (CytoSelect 96-Well Cell Transformation Assay kit, Cell Biolabs, Inc. San Diego, CA, USA) was also used to confirm the quantitative data. These results indicate that overexpression of wild-type YAP or constitutive active YAP in FT194 and FT246 cells stimulates colony formation, which is indicated by a significant increase $(P<0.001)$ in the relative fluorescence units in FT194-YAP, FT246-YAP, FT194-YAP ${ }^{\text {S127A }}$ and FT246-YAP ${ }^{\text {S127A }}$ cells (Figure 3).

YAP-transformed FTSECs forms tumor in vivo

The colony formation assays suggest that YAP is sufficient to transform immortalized FTSECs in vitro. In turn, we then examined 
a

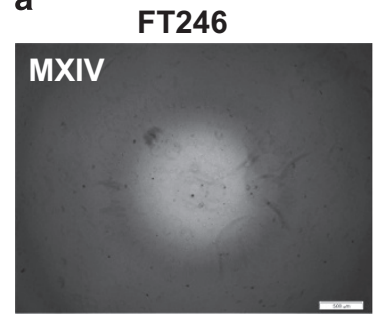

FT246

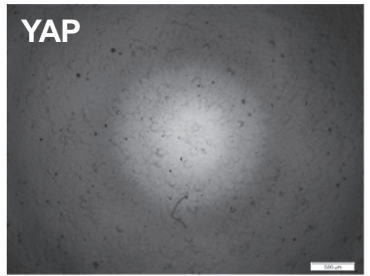

FT246

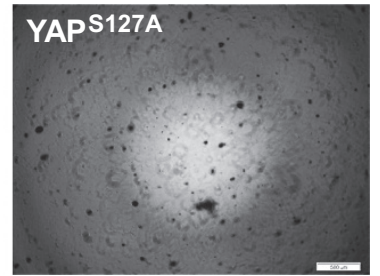

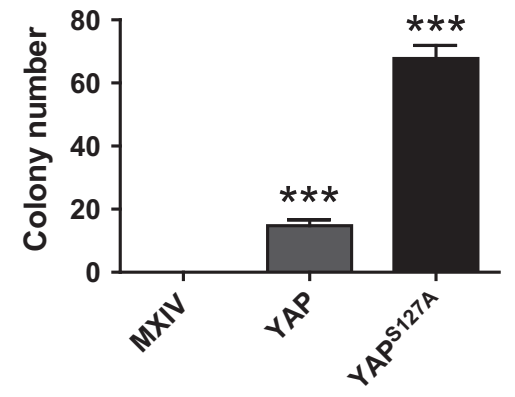

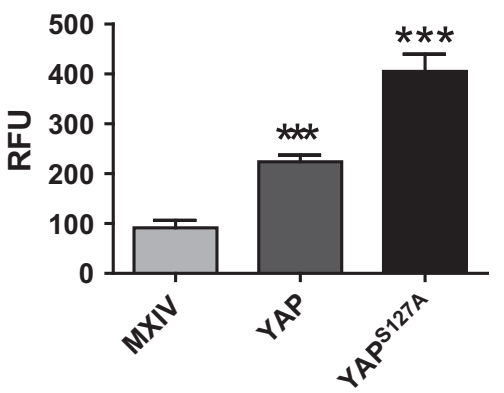

b
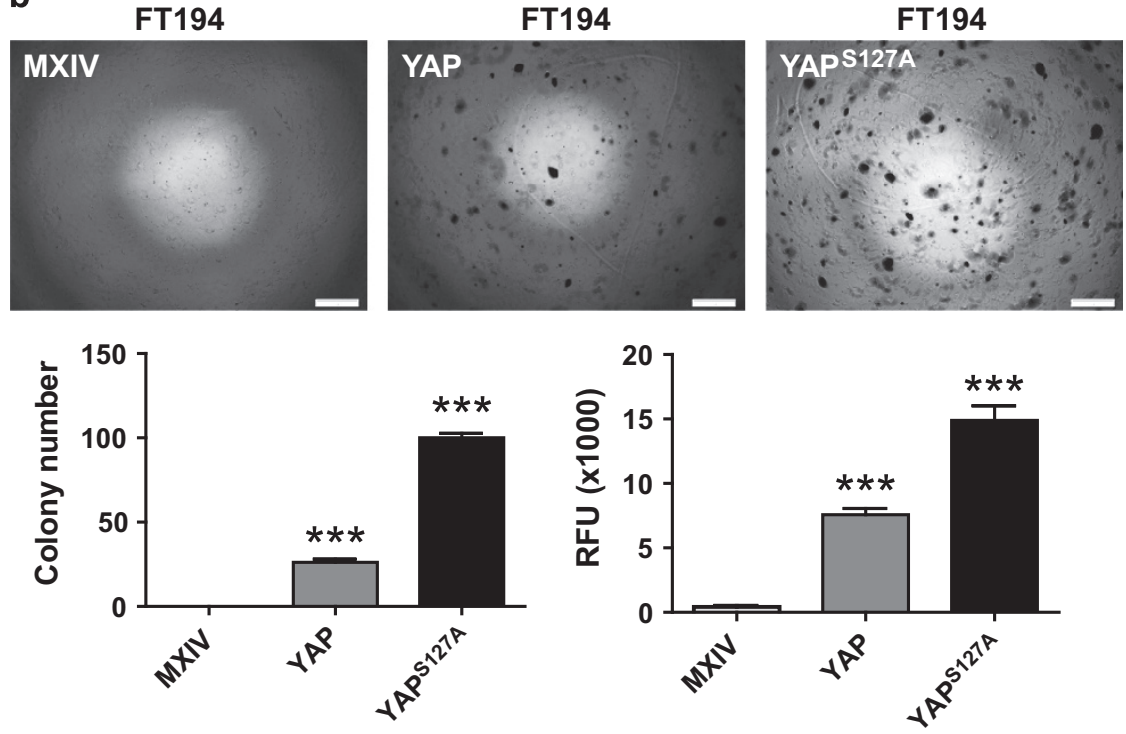

Figure 3. YAP is able to transform immortalized FTSECs. (a) Top panel: soft agar assay showing colony formation in FT246-MXIV, FT246-YAP and FT246-YAP ${ }^{\text {S127A }}$ cells. Scale bar $=500 \mu \mathrm{m}$. Lower panel: quantitative analysis of colony formation in FT246-MXIV, FT246-YAP and FT246$\mathrm{YAP}^{\mathrm{S} 127 \mathrm{~A}}$ cells. Left: colony numbers in FT246-MXIV, FT246-YAP and FT246-YAP ${ }^{\mathrm{S} 127 \mathrm{~A}}$ cells. Colonies with more than fifty cells were considered viable and counted. Right: fluorescence-based quantitative soft agar assay showing the relative colony numbers in FT246-MXIV, FT246-YAP and FT246-YAP ${ }^{\mathrm{S127A}}$ cells. (b) Soft agar assay showing colony formation in FT194-MXIV, FT194-YAP and FT194-YAP ${ }^{\text {S127A }}$ cells. Scale $\mathrm{bar}=500 \mu \mathrm{m}$. Lower panels are quantitative data of regular soft agar assays and the fluorescence-based soft agar assays. Each bar represents mean \pm s.e.m. of five assays. ${ }^{* * *} P<0.001$ compared with MXIV control.

if YAP-transformed cells are tumorigenic. FT194-MXIV, FT194-YAP and FT194-YAP ${ }^{\mathrm{S127A}}$ cells $\left(6 \times 10^{6}\right.$ cells per group) were injected SC into 5-week-old female athymic nude mice. Tumors were

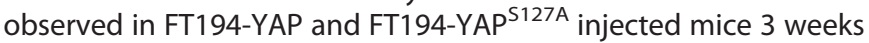
after injection. Consistent with colony formation data, no tumors were observed in the FT194-MXIV cell injected group (Figures 4a and b). Tumors derived from FT194-YAP ${ }^{5127 A}$ cells grew very rapidly. Tumors derived from FT194-YAP cells grew relatively slowly within the 10-week period (Figures $4 c$ and d).

Morphologically, tumors derived from the FT194-YAP and FT194-YAP ${ }^{\text {S127A }}$ cells resembled HGSC, possessing tumor cells that are generally intermediate to large in size, with prominent nucleoli visible at low magnification (Figure 5a). The nuclei are distinctly pleomorphic, showing more than a threefold variation in size. Similarly, these cells are extremely proliferative, which is indicated by the high expression of Ki-67 and frequent appearance of mitotic figures (Figure 5a). ${ }^{32}$ Except for the high levels of YAP protein, these tumor tissues have very high levels of nuclear stained of TP53, PAX8 and WT-1 (Figures 5a and 4e), which are characteristic of both FTSECs and the vast majority of HGSCs, but not ovarian surface epithelial-derived tumor or low-grade serous carcinomas. ${ }^{33-35}$ Both biochemical and immunohistochemical analysis show that tumor cells in these tissues express very high levels of cytokeratin 7, but are negative for cytokeratin 20 and PAX2 (Figures 5a and 4e). Tumor tissues derived from FT194YAP cells are negative for Alcian blue ( $\mathrm{pH} 2.5)$-periodic acid-Schiff staining (Figure $5 \mathrm{~b}$ ). These findings further confirm pathological diagnoses that these tumors are HGSC. ${ }^{36,37}$ 
YAP stimulates migration of FTSECs in vitro

Wound-healing assays show that, compared with the controls, overexpression of wild-type YAP or constitutively active YAP significantly enhances wound closure in FT246 (Supplementary Figure 8) and FT194 cells (Supplementary Figure 9). Transwell migration assay also indicate that ectopic expression

a

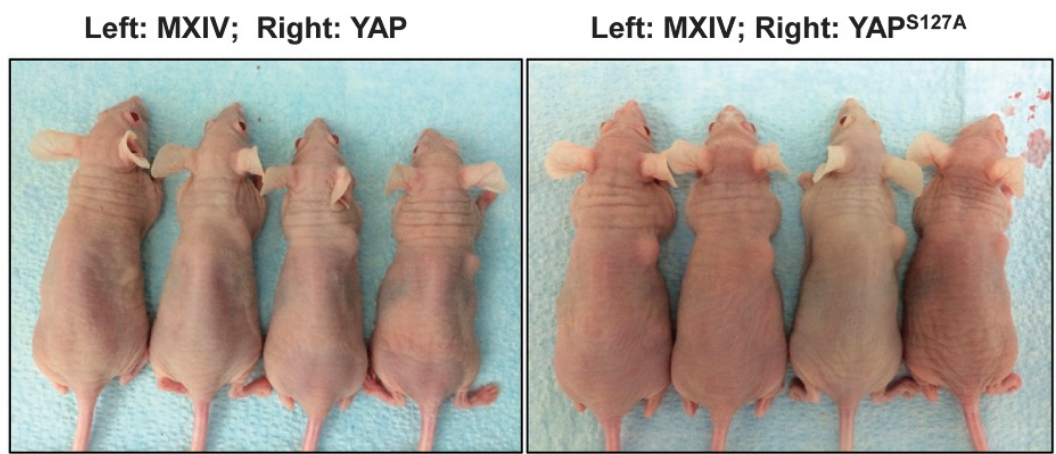

b
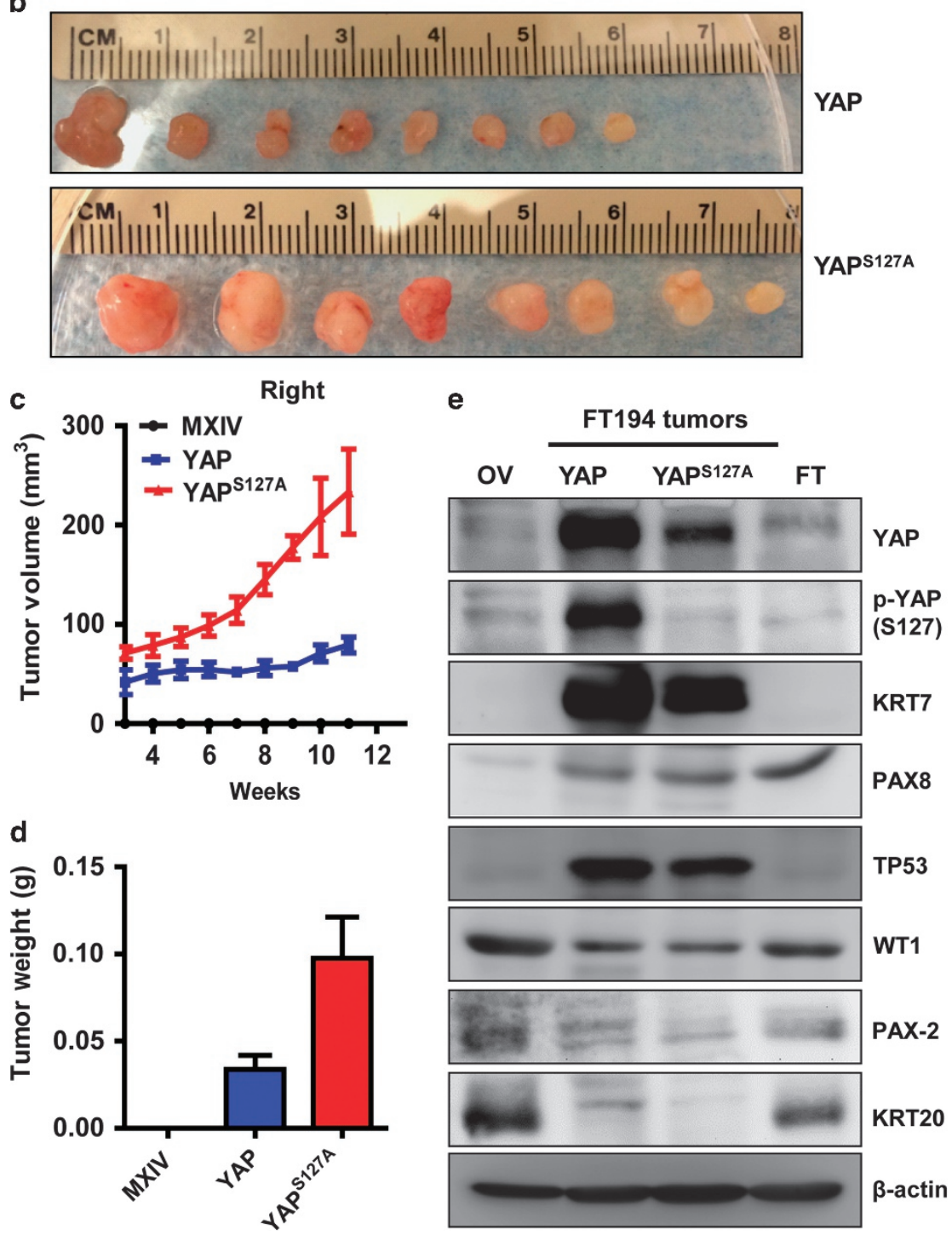

Figure 4. Tumorigenic effect of YAP in FTSECs. (a) Representative images showing tumor formation in the athymic nude mice injected with FT194-YAP and FT194-YAP ${ }^{\text {S127A }}$ cells. FT194-MXIV control cells were injected on the left side and did not form tumor. (b) Tumors derived from FT194-YAP and FT194-YAP ${ }^{\text {S127A }}$ cells. (c) Growth curves of tumors derived from FT194-YAP and FT194-YAP ${ }^{\text {S127A }}$ cells. (d) Weights of tumors derived from FT194-YAP and FT194-YAP ${ }^{\text {S127A }}$ cells. (e) Western blot analysis showing biomarkers expressed in tumors derived from FT194-YAP and FT194-YAP ${ }^{\text {S127A }}$ cells. Tumors derived from FT194-YAP and FT194-YAP ${ }^{\text {S127A }}$ cells express high levels of cytokeratin 7 (KRT7), PAX8, TP53 and WT-1, but very low cytokeratin 20 (KRT20) and PAX2. Same amount of proteins from normal human ovarian (OV) and fallopian tube (FT) tissues were used as control. 
of wild-type YAP or constitutively active YAP significantly increases the number of cells migrated through the chamber membrane in both FT246 (Supplementary Figure 8) and FT194 cells (Supplementary Figure 9).

YAP overexpression upregulates FGF ligands and FGFRs in FTSECs Previous reports show that isolated FTSECs can secret acidic (FGF1) and basic FGF2. ${ }^{23}$ FGF1 and FGF2, which are the most abundant FGF ligands in ovarian cancer cell lines, ${ }^{38}$ and all FGFRs (FGFR1, FGFR2, FGFR3 and FGFR4) were expressed in five immortalized human fallopian tube cell lines (Supplementary Figure 10). Of considerable interest, we found that overexpression of wild-type YAP or constitutively active YAP in FT194 and FT246 cells significantly increased mRNA levels of FGF1, FGF2 and all four FGFRs (Figure 6a, Supplementary Figures 11 and 12). Importantly, overexpression of wild-type YAP or constitutive active YAP in FT194 cells significantly increased protein levels of FGF2 (Figure 6b) and FGF1 (Figure 6c) in culture medium and on-dish cell lysates. Intriguingly, treatment of these cells with verteporfin, $^{39}$ a YAP antagonist, eliminated YAP- or YAP ${ }^{5127 A}$ induced production of FGF1 and FGF2 (Figures $6 \mathrm{~b}$ and c). Overexpression of wild-type YAP or constitutively active YAP in FT194 and FT246 cells also significantly increased the expression of amphiregulin (AREG) (Figure 6a, Supplementary Figures 11 and 12), which is a known downstream gene of the Hippo/YAP pathway. $^{40}$

FGF2 induces proliferation and migration of FTSECs

To determine the role of FGF1/2 in FTSECs, FT194 and FT246 cells were treated with different concentrations of recombinant human FGF1 and FGF2 $(10-100 \mathrm{ng} / \mathrm{ml})$ for $72 \mathrm{~h}$. FGF1/2 treatment significantly increased proliferation of FT194 and FT246 cells at a concentration of $10 \mathrm{ng} / \mathrm{ml}$ (Figures 7a and b). As FGF2 has been reported to be secreted by FTSECs ${ }^{23}$ and the biological activity of FGF2 is not significantly dependent on heparin, in the following experiments, we used FGF2 as a primary ligand in the study. The maximal pro-proliferative effect of FGF2 was observed at $10-20 \mathrm{ng} / \mathrm{ml}$ in both FT194 and FT246 cells. Flow cytometry results showed that FGF2 $(20 \mathrm{ng} / \mathrm{ml})$ treatment promoted cell cycle progression in FT194 and FT246 cells, as indicated by a significant decrease in the portion of cells in G1 phase and significant increase in cells in $S$ and $\mathrm{G} 2 / \mathrm{M}$ phases $(P<0.05$; Supplementary Figure 13). In addition, wound-healing assays showed that FGF2 treatment $(20 \mathrm{ng} / \mathrm{ml}, 15 \mathrm{~h})$ significantly induced wound closure in FT246 (Figure 7c) and FT194 cells (Figure 7d), suggesting that FGF2 also induces FTSECs migration.

YAP is required for FGF regulating proliferation and migration of FTSECS

To determine if YAP has a role in FGF2-stimulated proliferation of FTSEC, we knocked down YAP protein in FT194 and FT246 cells using YAP siRNAs and then treated these cells with FGF2. Results showed that FGF2 failed to promote the proliferation of FT194 and FT246 cells after knockdown of YAP (Figure 8a). Flow cytometry results showed that knockdown of YAP completely blocked FGFpromoted cell cycle progression in both FT194 (Supplementary Figure 14a) and FT246 cells (Supplementary Figure 14b). Knockdown of YAP not only blocked FGF2-induced cell proliferation, but also reduced basal growth of FT194 and FT246 cells, suggesting that YAP was required for the survival of FTSECs (Figure 8a). In addition, we found that knockdown of YAP in FTSEC cells diminished FGF2-stimulated cell migration, as indicated by the significant decrease in the wound closure in YAP knockdown FT194 and FT246 cells after FGF2 treatment (Figure 8b, Supplementary Figure 15). Interestingly, FGF2 treatment $(10 \mathrm{ng} / \mathrm{ml}$, $48 \mathrm{~h}$ ) induced AREG mRNA expression in both FT194 and FT246 cells, but had no effect on YAP mRNA expression. Knockdown of YAP completely blocked FGF2-induced AREG mRNA expression in both FT246 and FT194 cells (Figure 8c). These results clearly indicate that YAP protein is required for FGF to regulate FTSEC cell proliferation, migration and gene expression. Intriguingly, we found that FGF2 was able to stimulate FGF1, FGF2 mRNA expression in cultured FTSECs, indicating that FGF2 may regulate the immortalized FTSECs in an autocrine and/or paracrine manner (Figure 8d, Supplementary Figure 16). Knockdown of YAP blocked FGF2-induced expression of FGF1/2, suggesting that the proper interaction between the Hippo/YAP and FGF/FGFR signaling pathways are also required for the maintenance of the autocrine/paracrine regulation of FTSECs by the FGF/FGFR system (Figure 8d, Supplementary Figure 16).

The Hippo pathway is involved in YAP regulation of FTSEC cell activities

The production of FGF ligands and FGFRs in FTSEC cells suggests that an autocrine and/or paracrine mechanism exists in FTSECs to regulate cell activities. Treatment of FT194 cells with $20 \mathrm{ng} / \mathrm{ml}$ of FGF2 rapidly suppressed phosphorylation LATS1 at both threonine 1079 and serine 909 (Figure 9a, Supplementary Figure 17). Further, treatment with FGF2 suppressed phosphorylation of Mob1 at threonine 35 and YAP at serine 127. FGF2 treatment had no effect on the total protein levels of these core components of the Hippo pathway signaling cascade (Figure 9a, Supplementary Figure 17). These pieces of evidence suggest that the Hippo pathway is involved in the YAP and FGF interaction, despite of the fact that YAP can be activated in a Hippo-independent manner. ${ }^{41}$

As mentioned above, LATS1/2 are the major suppressors of YAP activity in the Hippo/YAP pathway. ${ }^{13,16}$ Consistent with above observations, knockdown of LATS1/2 in FT194 cells with LATS1/2 siRNAs activated YAP (S127), which is indicated by significantly decrease of YAP phosphorylation at serine 127 (Figure 9b, Supplementary Figure 18). Knockdown of LATS1/2 had no effect on the total YAP protein level (Figure 9b, Supplementary Figure 18). As expected, knockdown of LATS1/2 significantly increased FGF1 $(P<0.01)$, FGF2 $(P<0.05)$, FGFR1 $(P<0.01)$ and FGFR4 $(P<0.01)$ expression in FT194 cells (Figure 9c, Supplementary Figure 19). Importantly, inhibition of YAP or FGFR activities with verteporfin or BGJ398, respectively, not only eliminated increases in cell proliferation and migration induced by LATS knockdown (Figures 9d and e, Supplementary Figure 20), but also reduced the basal-level growth of FT194 cells (Figure 9d).

FGF treatment also rapidly (within $10 \mathrm{~min}$ ) activated the Raf/ extracellular signal-regulated kinase (ERK) and the phosphatidylinositol 3 kinase (PI3K)/AKT pathways (Figure 9a), which have been shown to be activated by binding of FGF2 to FGFRs. ${ }^{42}$ Knockdown of LATS1/2 also increased phosphorylation of AKT (S473) and ERK1/2 (T202/Y204) (Figure 9b). Further, FGF2-induced suppression of YAP phosphorylation was blocked by the FGFR inhibitor BGJ398, PI3K inhibitor LY294002 and by MEK inhibitor UO126, suggesting that the $\mathrm{PI} 3 \mathrm{~K}$ and mitogen-activated protein kinase (MAPK) pathways are involved in FGF/FGFR-induced suppression of Hippo/YAP signaling in FTSECs (Supplementary Figure 21a). In a hanging drop culture system, treatment of FT194 cells with LY294002 (PI3K inhibitor) completely inhibited FTSEC growth, leading to disassembly of the three-dimensional (3D) structure. However, treatment with U0126 only partially inhibited YAPinduced FTSEC cell growth in the 3D culture system. This indicates that PI3K and MAPK pathways are involved in YAP regulation of FTSEC growth, but potentially in differential ways (Supplementary Figure 21b). 
a
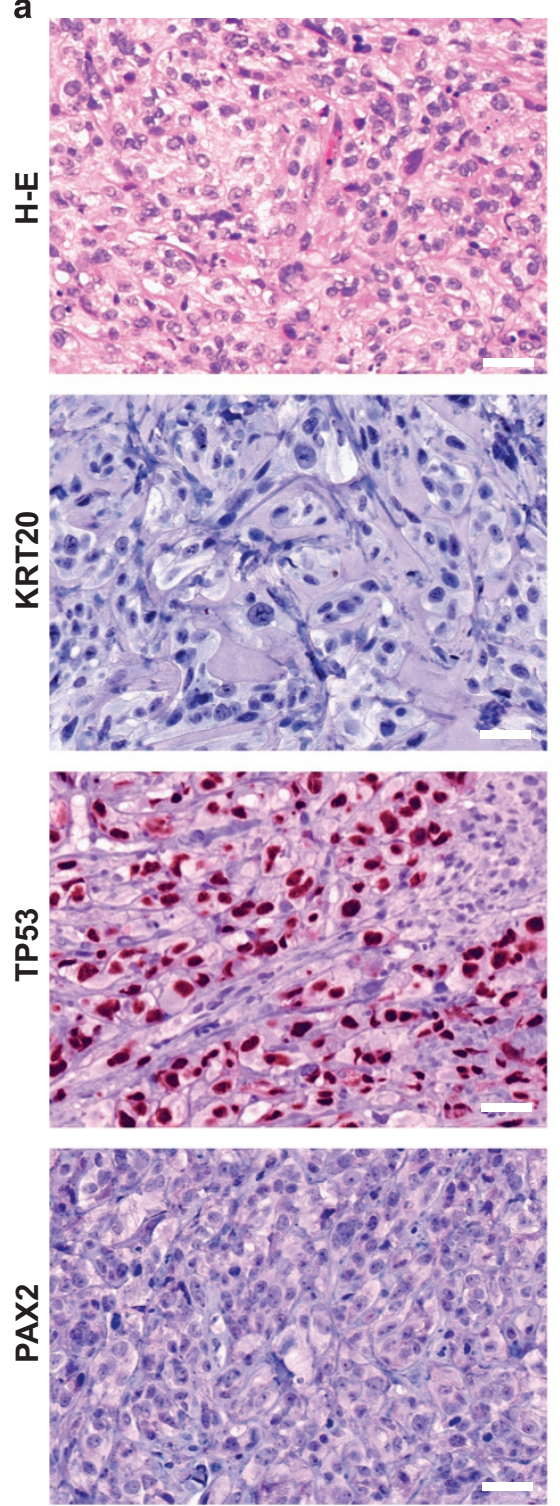

b

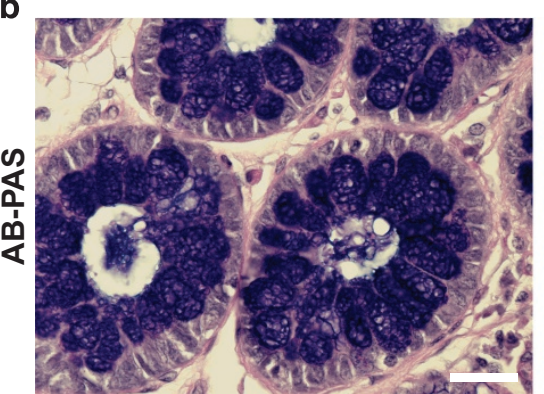

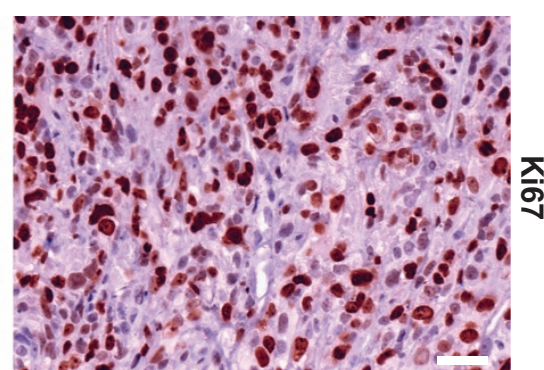
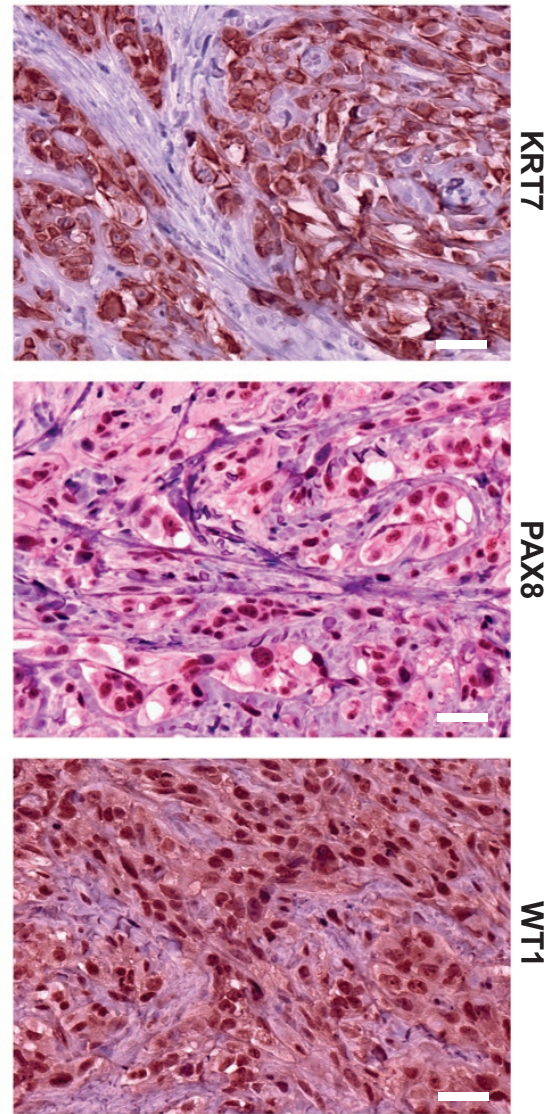

$\xi$

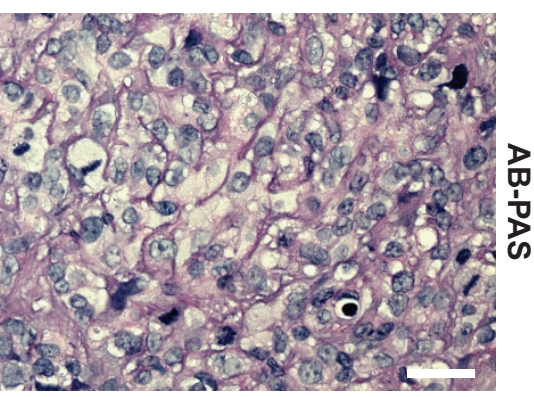

Figure 5. YAP induces fallopian tube HGSC. (a) Histology and molecular features of tumors derived from FT194-YAPS127A cells. H-E: representative image showing histology of FT194-YAP ${ }^{\text {S127A }}$ tumors tissues stained with hematoxylin-eosin. Ki-67: representative image showing expression of Ki-67 protein in FT194-YAP ${ }^{\text {127A }}$ tumor tissues analyzed by immunohistochemistry. FT194-YAP ${ }^{5127 A}$ tumors also express high level of cytokeratin 7 (KRT7), nuclear TP53, PAX8 and WT-1, but not cytokeratin 20 (KRT20) and PAX2. Scale bar: $20 \mu \mathrm{m}$. (b) Representative images showing that tumor tissues derived from FT194-YAP cells are negative for the Alcian blue pH 2.5-periodic acid-Schiff (AB-PAS) staining. Left: colorectal epithelium was used as a positive control for AB-PAS staining. Right: tumor tissues derived from FT194-YAP cells were AB-PAS negatively stained. Scale bar: $25 \mu \mathrm{m}$. 
The Hippo/YAP pathway interacts with FGF pathway to regulate fallopian tube cell growth

To examine whether FGFR signaling pathway is required by YAP to regulate FTSEC cell activities, we knocked down FGFRs with four FGFR siRNAs (Supplementary Figure 22). Knockdown of FGFRs inhibited YAP-induced or constitutively active YAP-induced growth of FTSECs (Figure 10a, Supplementary Figure 22). The same results was observed if BGJ was used to block FGFRs (Figure 10a, Supplementary Figure 22). Wound-healing assay showed that treatment of FT194 cells with BGJ or verteporfin significantly blocked 'wound' closure in FTSECs, suggesting that these pathways are important for the migration of FTSECs (Supplementary Figure 23). Importantly, blockage of FGFRs with BGJ eliminates YAP-induced or constitutively active YAP-induced anchorage-free growth of FTSECs, suggesting that FGFR signaling pathway is also involved in the transformation of FTSECs (Figures $10 \mathrm{c}$ and $\mathrm{d}$ ). OVSAHO is a verified ovarian HGSC cell line. ${ }^{43}$ Treatment of OVSAHO cells with BGJ and verteporfin also eliminated its ability of anchorage-free survival (Supplementary Figure 24), further confirming the critical role of FGFR in YAP-induced carcinogenesis of FTSECs.

Previous studies has shown that spheroid formation is one of the most well-characterized models for 3D culture and screening because of its simplicity, reproducibility and similarity to physiological tissues. ${ }^{44}$ We then used a hanging drop culture system to further examine the biological function of the interaction between the Hippo/YAP and the FGFR signaling pathways in FTSECs. We found that ectopic expression of wildtype YAP or constitutively active YAP in FT194 cells significantly induced cell growth in the 3D culture system (Figure 10b). YAP-induced growth of FTSECs in a 3D hanging drop culture system was significantly suppressed by verteporfin (YAP antagonist) $^{39}$ and BGJ398 (FGFR inhibitor) ${ }^{25}$ (Figure 10b). In the BGJ-treated FT194 cells, we observed that BGI398 blocked YAP and YAP ${ }^{\text {127A }}$-induced FTSEC growth (indicated by the smaller spheroids in groups; Figure 10b). In the verteporfin-treated FT194 cells, the spheroids did not shrink, but we clearly observed that well-defined edges of these spheroids disappeared. The cell-cell contacts in these drug-treated spheroids are so loose that these cells can be easily dispersed by pipetting. Staining of Ki-67 indicated that both verteporfin and BGJ398 treatments suppressed YAP- and YAP ${ }^{\mathrm{S} 127 \mathrm{~A}}$-induced Ki-67 expression, suggesting that the Hippo/YAP pathway and FGFR signaling pathway are critical for the cell-cell communication and growth of the FTSECs (Supplementary Figure 25). Terminal deoxynucleotidyl transferase-mediated dUTP nick end labeling (TUNEL) assay showed that treatment of FTSECs with verteporfin and BGJ398 markedly increased TUNEL-positive cells, further supporting the role of the Hippo/YAP signaling pathway and the FGFR pathway in the viability of FTSECs (Supplementary Figure 26).

Finally, we use a xenograft tumor tissue culture system to examine the role of FGFR signaling pathway and oncogene YAP on the growth of xenograft tumor cells. Treatment of tumor tissues for 3 days suppressed tumor cell growth and induced tumor cell death, which was indicated by the decreased Ki-67positive cells and increased TUNEL-positive cells in the tumors derived from FT194-YAP ${ }^{\text {S127A }}$ cells (Figure 10e). Similar results were also observed in tumors derived from OVSAHO cells, a cell line that has been verified as ovarian HGSC cells, ${ }^{43}$ after treatment for 3 days with antagonists against FGFRs and YAP (Supplementary Figure 27). These lines of evidence clearly suggest that the proper interaction between the Hippo/YAP and FGF/FGFR signaling pathways are not only essential for FTSECs growth, but also for FTSEC transformation and potentially fallopian tube and ovarian HGSC initiation.

\section{DISCUSSION}

FTC has long been considered as a rare type of gynecological cancer. ${ }^{11,45}$ However, the present criteria for diagnosis and classification of FTC are not well established. Presently, pelvic cancers are classified as FTC only in the presence of a dominant tubal mass and a precursor lesion in the fallopian tube, with no mass observed in the ovary or endometrium. ${ }^{10}$ Similarly, primary peritoneal cancer is diagnosed only when no mass is found in the ovary or the fallopian tube. Interestingly, the presence of a precursor lesion in the ovary is not a requirement to diagnose a tumor of ovarian origin. ${ }^{46}$ Furthermore, the convention has been to classify serous tumors in the pelvis as ovarian cancer when the origin is unclear. ${ }^{8}$ Owing to the fact that many cases of FTC also have tumors on the surface of the ovary, ${ }^{8-11,46,47}$ the previous criteria for diagnosis and classification of FTC, primary peritoneal cancer, and ovarian cancer have led to significant underestimation of the incidence of FTCs. Recent studies identify the FTSECs as the cell origin of ovarian HGSC provide direct evidence for the importance of FTC in the initiation and progression of the conventional ovarian HGSC. ${ }^{9,48}$

Despite progress in identifying the FTSEC origin of ovarian HGSC, the mechanism underlying the initiation and progression of fallopian tube and ovarian HGSCs remains unclear. Previous studies show that YAP overexpression occurs in a broad range of human cancers, ${ }^{49-51}$ including ovarian cancer, ${ }^{17,18,20,52}$ and has been identified as an oncogene, ${ }^{13,16}$ although several reports indicate that YAP also functions as a tumor suppressor in certain types of cancer. ${ }^{53,54}$ Our recent data show that YAP is overexpressed in the ovarian adult granulosa cell tumor and has critical roles in regulating granulosa cell tumor cell proliferation, migration and steroidogenesis. ${ }^{19}$ We also found that YAP interacts with epidermal growth factor receptor signaling pathway to regulate growth of ovarian surface epithelial cells and ovarian cancer cells. ${ }^{20}$ In this study, we found that in the normal fallopian tube cells, YAP expression was very low and was primarily located in the cytoplasm of the ciliated epithelial cells. However, YAP was overexpressed and localized in the nucleus of almost all epithelial cells in the inflammatory and cancerous fallopian tube tissues. These results suggest that the Hippo/YAP pathway may be involved in the initiation and progression of fallopian tube epithelia-derived cancer. This hypothesis is supported by observations that: (1) overexpression of wild-type YAP or constitutively active YAP induces FTSEC proliferation, whereas knockdown of YAP results in suppression of FTSEC proliferation; (2) ectopic expression of wild-type YAP or constitutively active YAP-induced transformation of FTSECs; and (3) FTSECs transformed by wild-type YAP or constitutively active YAP are tumorigenic, as indicated by the formation and growth of tumor xenograft in athymic nude mice. This is the first report to show that YAP has a critical role in initiating FTC. Owing to emerging evidence that ovarian HGSC is primarily derived from transformed FTSECs, ${ }^{8-11}$ it is reasonable to theorize that YAP has critical roles in the development of ovarian HGSC. Results from analysis of the multidimensional genomics data indicate that YAP and TEAD genes are frequently amplified and upregulated in ovarian HGSC (Figure 1f), whereas LATS1, the main component of the Hippo signaling pathway and the major negative regulator of YAP activity, is downregulated (Figure 1f). Results from these large-scale sequencing analyses not only support our hypothesis that the Hippo/YAP signaling pathway have critical role in the initiation and progression of fallopian tubederived ovarian HGSC, but also provide evidence for the clinical relevance of this study. As YAP activity depends on its expression level, phosphorylation status (that is, phosphorylation level and sites) and subcellular localization, upregulation of YAP mRNA or protein levels alone cannot reflect its activity, making it difficult to analyze the relationship between YAP activity and patient survival, especially in ovarian HGSC. Interestingly, we found that in the 418 


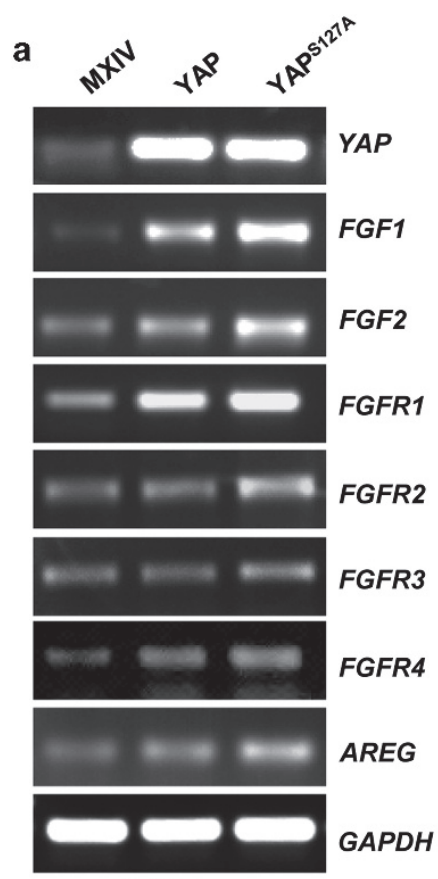

FT194

b

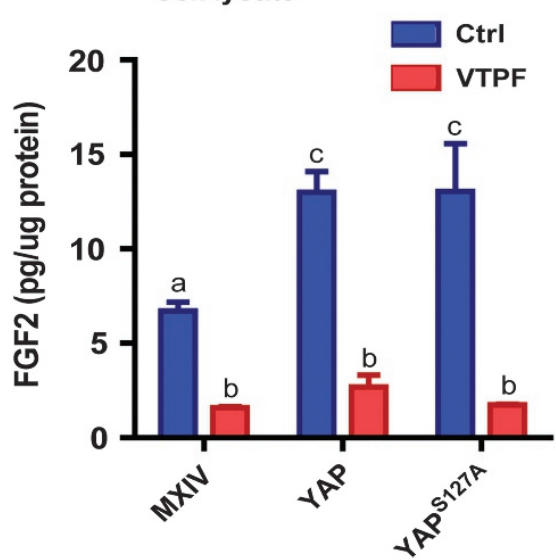

C

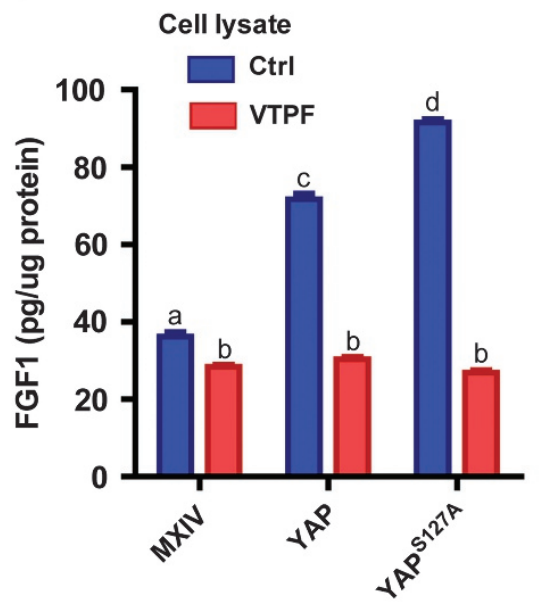

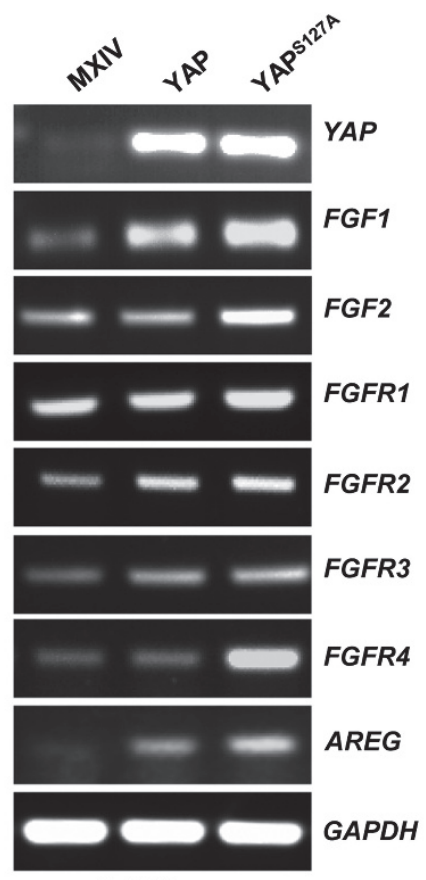

FT246

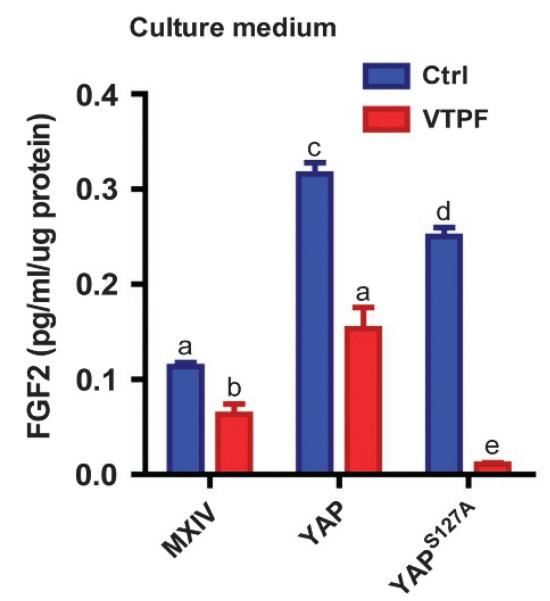

Culture medium

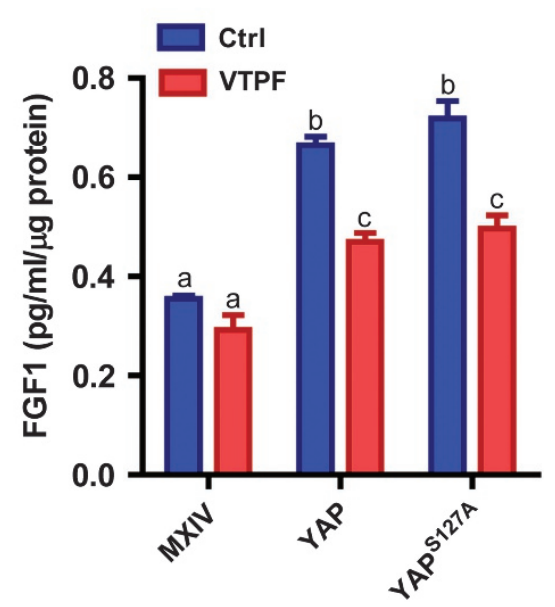


a

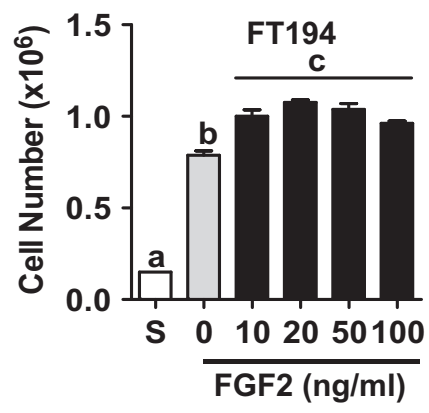

b

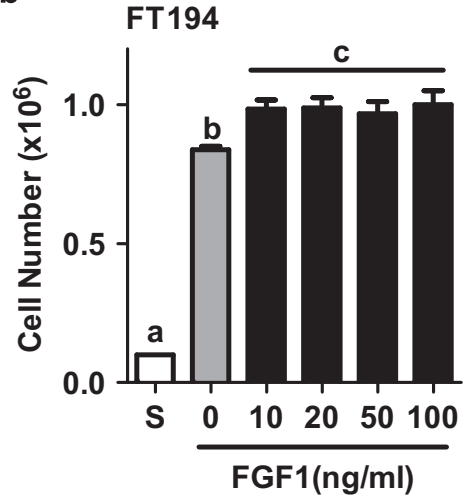

C

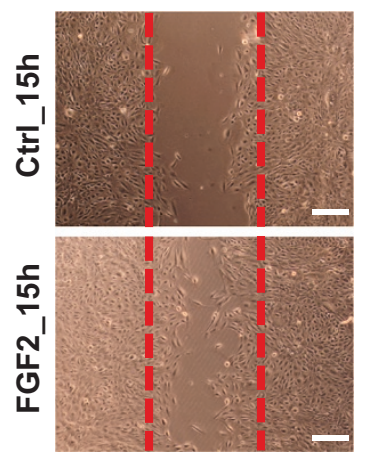

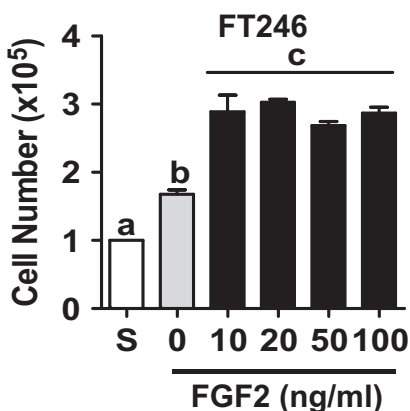

FT246

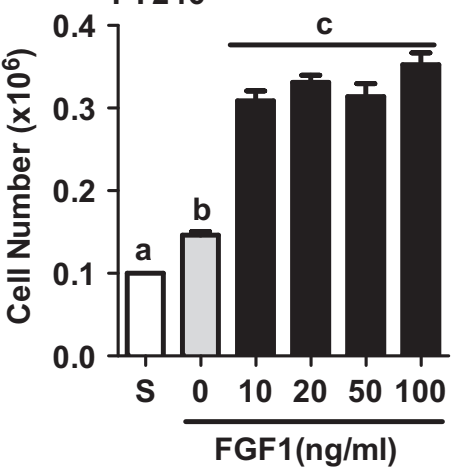

d

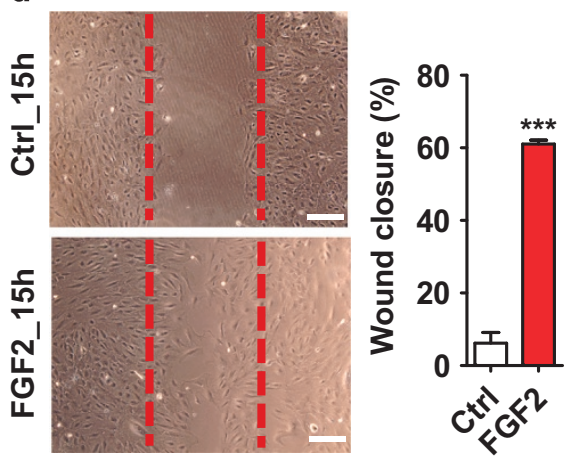

Figure 7. FGF stimulates the proliferation and migration of FTSECs. (a) Proliferation of FT194 (left panel) and FT246 (right panel) cells incubated in medium containing $1 \%$ fetal bovine serum (FBS) in the absence (0) or presence of 10, 20, 50 or $100 \mathrm{ng} / \mathrm{ml}$ basic FGF (FGF2) for 3 days. S, seeding cell number. Each bar represents the mean \pm s.e.m. of three repeats. Bars with different letters are significantly different from each other $(P<0.05)$. (b) Proliferation of FT194 (left panel) and FT246 (right panel) cells incubated in medium containing $1 \%$ FBS in the absence (0) or presence of $10,20,50$ or $100 \mathrm{ng} / \mathrm{ml}$ acidic FGF (FGF1) for 3 days. S, seeding cell number. Each bar represents the mean \pm s.e.m. of three repeats. Bars with different letters are significantly different from each other $(P<0.05)$. (c) Left panel: representative images showing the effect of FGF2 $(20 \mathrm{ng} / \mathrm{ml}, 15 \mathrm{~h})$ on the wound closure of FT194 cells. Right panel: quantitative data showing percentage of wound closure in FT194 cells in the absence or presence of $20 \mathrm{ng} / \mathrm{ml} \mathrm{FGF2.} \mathrm{(d)} \mathrm{Left} \mathrm{panel:} \mathrm{representative} \mathrm{images} \mathrm{showing} \mathrm{the} \mathrm{effect} \mathrm{of} \mathrm{FGF} \mathrm{treatment} \mathrm{(FGF2,}$ $20 \mathrm{ng} / \mathrm{ml}, 15 \mathrm{~h}$ ) on the wound closure of FT246 cells. Right panel: quantitative data showing percentage of wound closure in FT246 cells in the presence or absence of $20 \mathrm{ng} / \mathrm{ml} \mathrm{FGF2.} \mathrm{Each} \mathrm{bar} \mathrm{represents} \mathrm{means} \pm$ s.e.m. of three independent experiments. ${ }^{* * *} P<0.001$ compared with the control (Ctrl).

Figure 6. YAP stimulates expression FGF and FGFR and secretion of basic and acidic FGFs. (a) Determining the mRNA expression of $Y A P$, FGF ligands (FGF1\&FGF2) and FGFR1-4 in FT194-MXIV, FT194-YAP and FT194-YAP ${ }^{\text {S127A }}$ cells (left panel), and FT246-MXIV, FT246-YAP and FT246$Y_{\text {YAP }}{ }^{\text {127A }}$ cells (right panels) by RT-PCR. AREG was used as a positive control. GAPDH was used as a loading control. (b) Verteporfin (VTPF) suppresses YAP-induced production of basic FGF (FGF2). Left panel: FGF2 levels in the cell lysate of FT194-MXIV, FT194-YAP and FT194$\mathrm{YAP}^{\mathrm{S127A}}$ cells in the absence or presence of $5 \mu \mathrm{M}$ of VTPF. Right panel: FGF2 levels in the culture medium of FT194-MXIV, FT194-YAP and FT194-YAP ${ }^{5127 A}$ cells in the absence or presence of $5 \mu \mathrm{M}$ of VTPF. (c) Verteporfin (VTPF) suppresses YAP-induced production acidic FGF (FGF1). Left penal: FGF1 levels in the cell lysate of FT194-MXIV, FT194-YAP, and FT194-YAP $127 \mathrm{~A}$ cells in the absence or presence of 5 $\mu$ M of VTPF. Right panel: FGF1 levels in the culture medium of FT194-MXIV, FT194-YAP and FT194-YAP ${ }^{\text {S127A }}$ cells in the absence or presence of 5 $\mu$ M of VTPF. Each bar represents the mean \pm s.e.m. of at least three independent experiments. Bars with different letters are significantly different from each other $(P<0.05)$ 
a

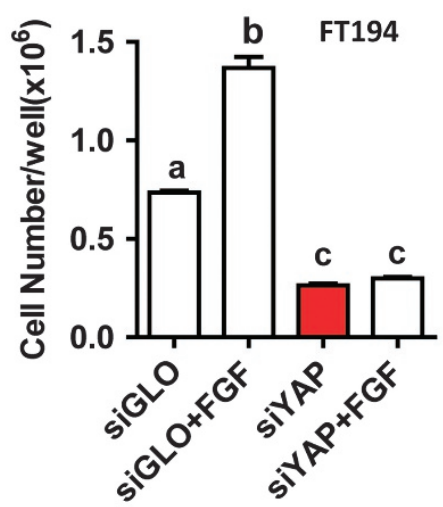

b
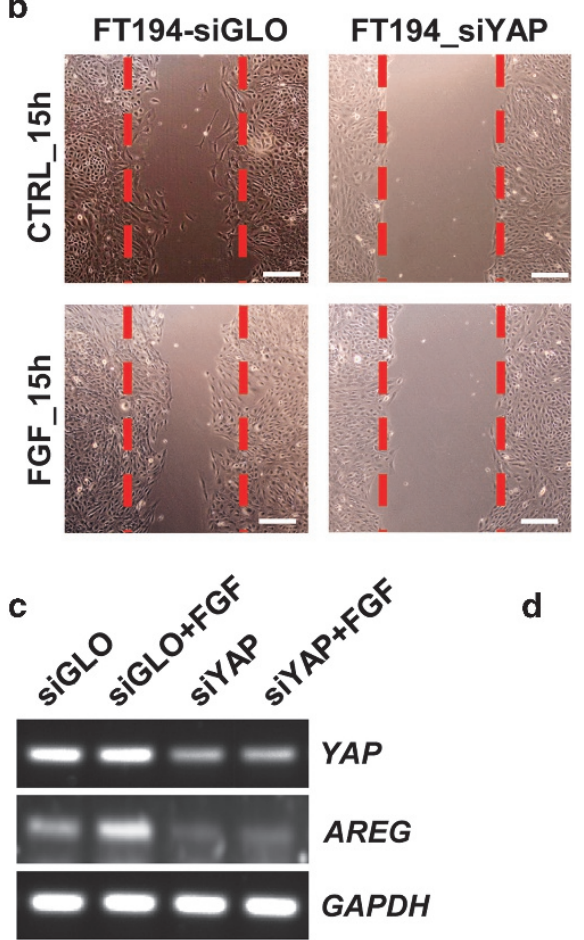

FT194
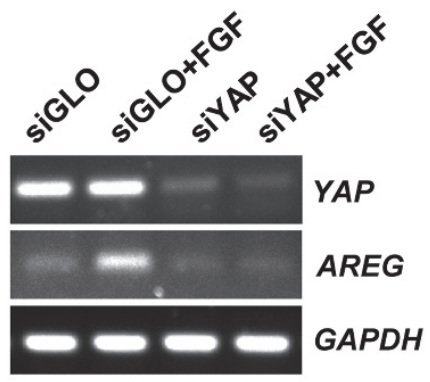

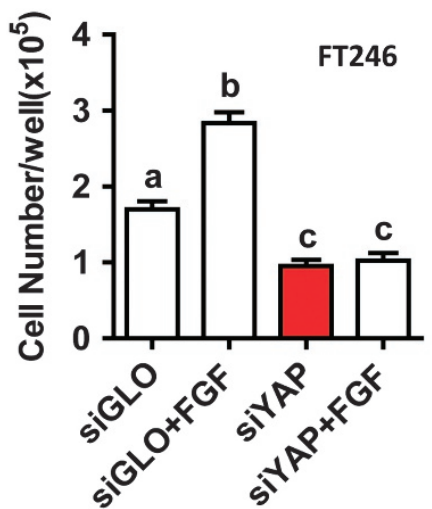

FT246 siGLO
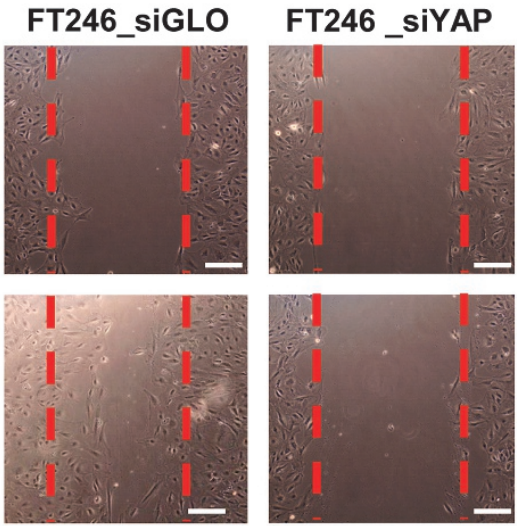

d

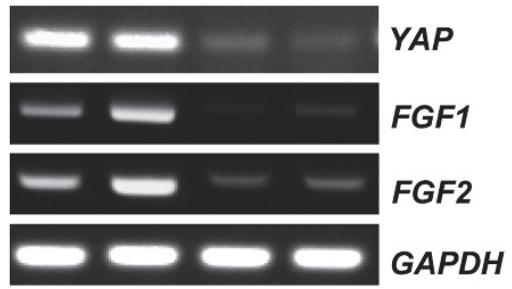

FT246

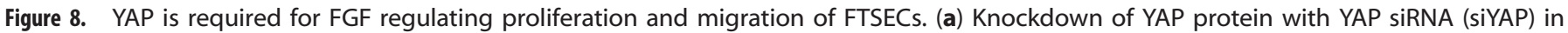

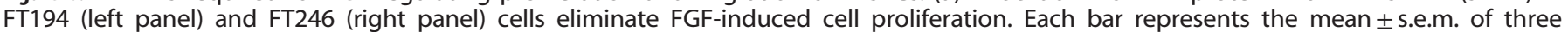

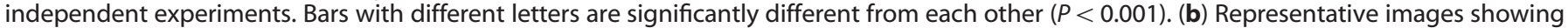

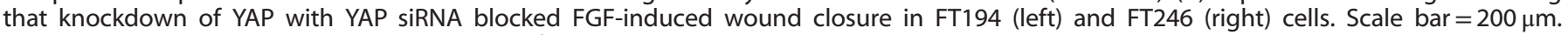

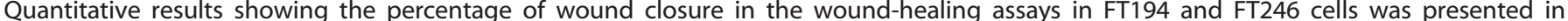

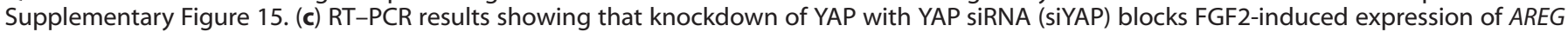

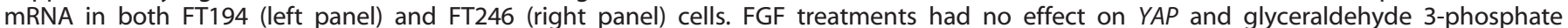

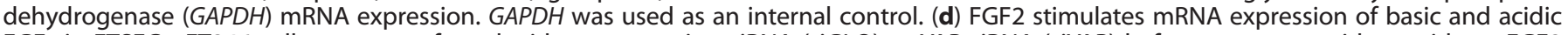

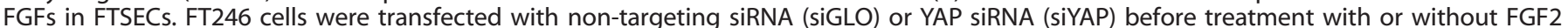

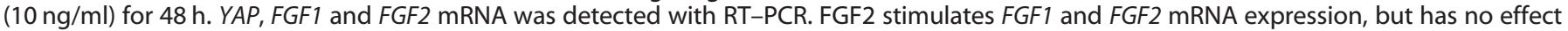
on $Y A P$ and GAPDH mRNA expression. GAPDH was used as an internal control. 
a

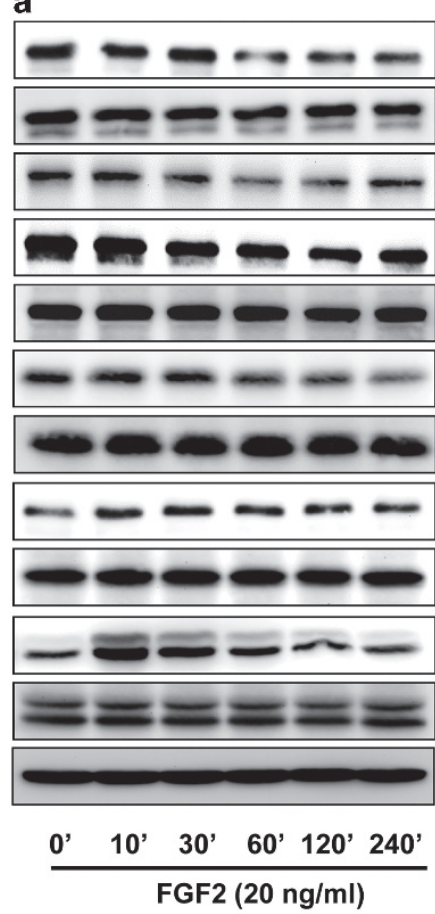

d

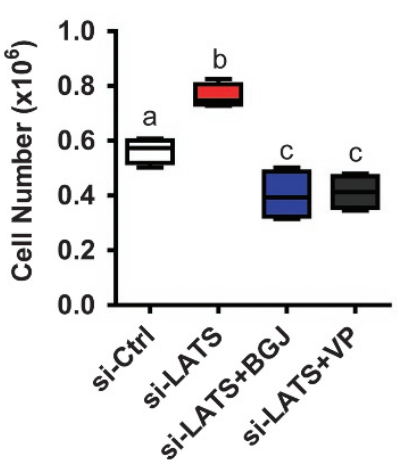

b

p-YAP
(S127)
YAP
p-LATS1
(T1079)
p-LATS1
(S909)
LATS1
p-MOB1
MOB1
p-AKT
AKT
p-ERK1/2
ERK1/2
$\beta-$ actin

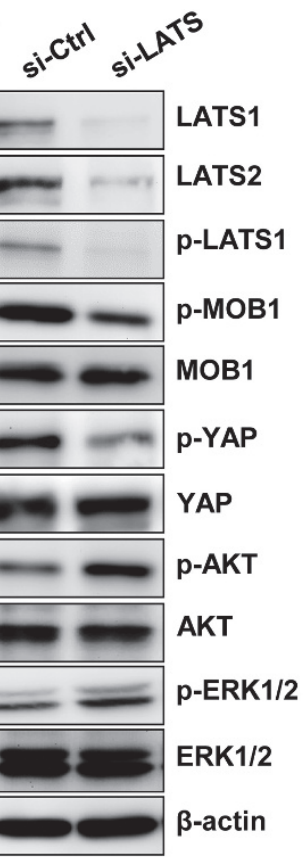

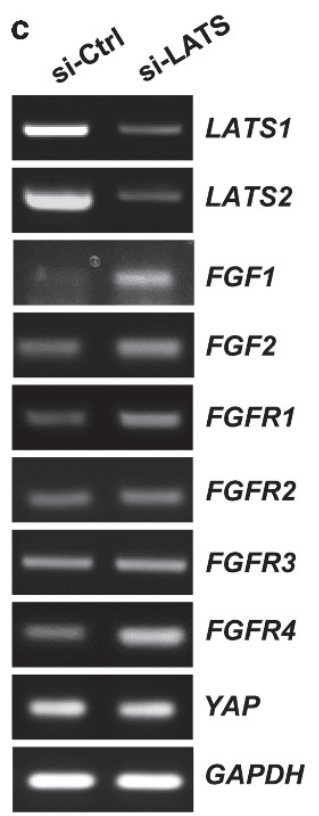

e

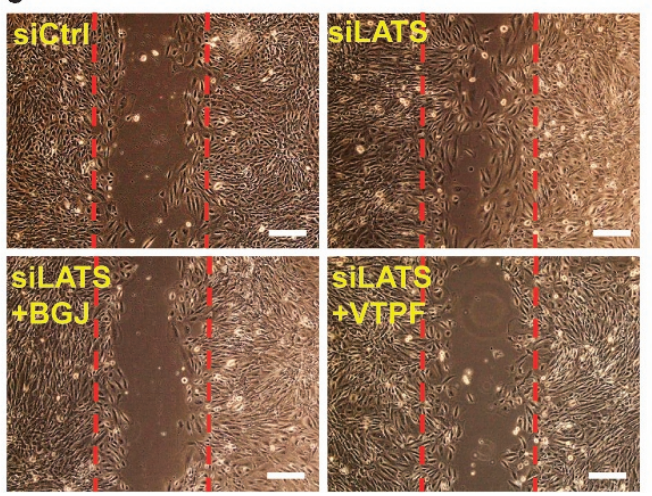

Figure 9. The Hippo pathway is actively involved in the interaction between YAP and FGFR signaling pathway in the FTSECs. (a) FGF2 suppresses the phosphorylation of the major components of the Hippo/YAP signaling pathways in FT194 cells. The total and phosphorylated proteins were determined by western blot. (b) Western blot analysis indicates that knockdown of LATS1/2 in FT194 cells using LATS1/2 specific siRNA activates YAP, AKT (S473) and ERK1/2. (c) RT-PCR results showing that knockdown of LATS1/2 in FT194 cells induces expression of FGF1, FGF2, FGFR1 and FGFR4. (d) Blockage of YAP and FGFR using verteporfin and BGJ398 inhibited FT194 cell proliferation induced by LATS1/2 knockdown. Each box represents mean \pm s.e.m. of four independent experiments. Boxes with different letters are significantly different from each other $(P<0.01)$. (e) Blockage of YAP and FGFR using verteporfin and BGJ398 inhibited FT194 cell migration induced by LATS1/2 knockdown. BGJ: BGJ398 $(1 \mu \mathrm{M})$; VTPF: verteporfin $(5 \mu \mathrm{m})$. Scale bar $=200 \mu \mathrm{m}$.

patients with ovarian HGSC, YAP and TEADs genes were significantly downregulated in 19 HGSC patients (beyond the default Z-score threshold). The survival analysis indicated that downregulation of YAP and TEADs in ovarian HGSC may be associated with improved patient survival (Figure 1g). However, larger samples from ovarian HGSC patients are needed to confirm this finding.

As noted in the introduction, the etiology of fallopian tube and ovarian HGSC is unknown. Indeed, several research groups have sought to identify the potential molecules or pathways that underlie FTSEC cell transformation and ovarian HGSC progression. The combination of SV40 T/t antigen and human telomerase reverse transcriptase (hTERT) expression resulted in immortalized FTSECs that were non-tumorigenic in mice. ${ }^{21,22}$ Jazaeri et al. ${ }^{21}$ showed that activation of HRAS G12V and C-MYC T58A in FTSECs harboring SV40 T and hTERT were the minimal alterations required to achieve transformation of human FTSECs. These results are consistent with observations by Kendall et al. who showed that forced activation of MYC, RAS ${ }^{\mathrm{v} 12}$ and hTERT, in addition to interference with TP53 and RB1 tumor-suppressor functions (mediated by SV40 T), were necessary and sufficient to transform human cells of epithelial and mesenchymal origin. ${ }^{55}$ On the other hand, studies by Karst et $a l^{22}$ indicate that introduction of $\mathrm{H}_{-\mathrm{RAS}} \mathrm{V}^{\mathrm{V} 2}$ or C-MYC transforms FTSECs and induces formation of HGSC. These results are supported by Shan et al. ${ }^{56}$ who also found that overexpression of HRAS $S^{V 12}$ transformed human fallopian tube epithelial cell lines that were immortalized with SV40 T/g antigens and hTERT. It is notable that in all three experiments, mutated HRAS has been used as an oncogene to transform the FTSECs. However, HRAS mutations are not characteristic of ovarian HGSC. ${ }^{57}$ 

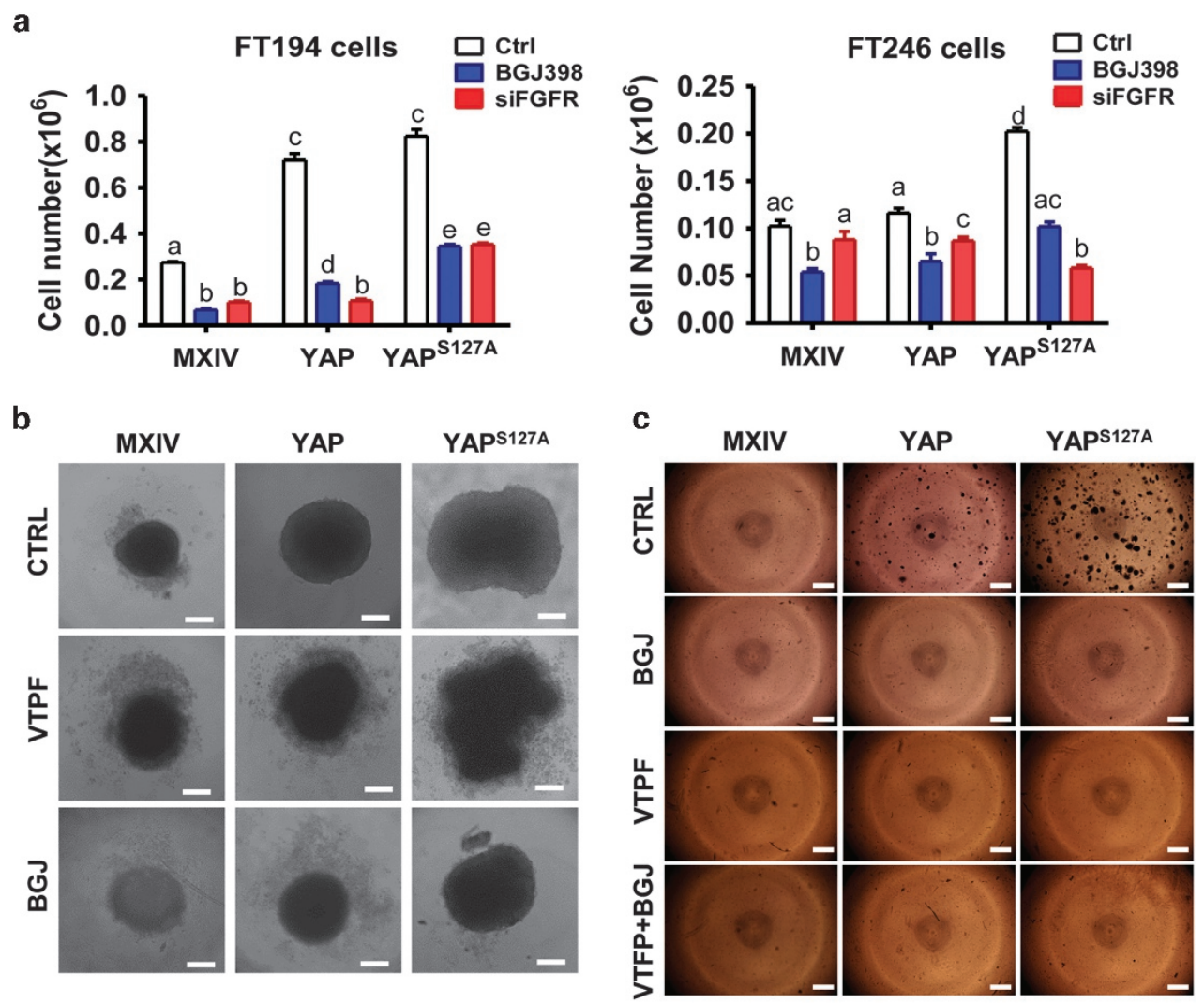

d

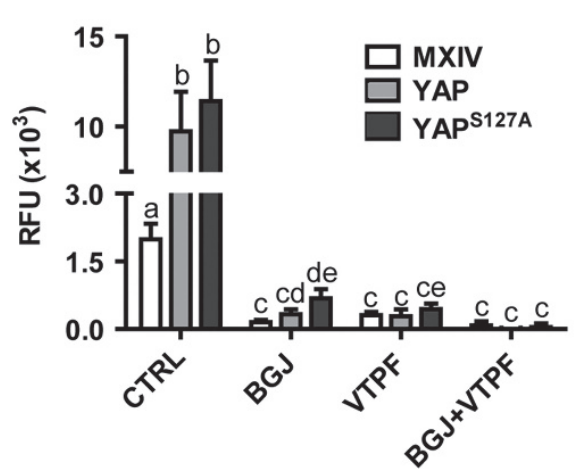

e

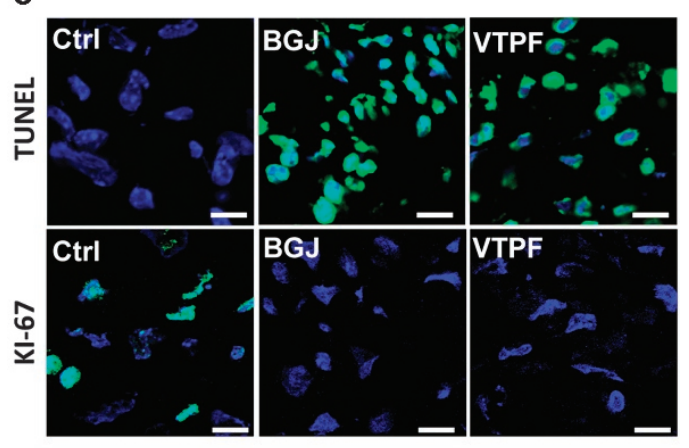

Figure 10. The Hippo/YAP pathway interacts with FGF/FGFR pathway to regulate activities of FTSECs. (a) Knockdown of FGFRs in FT194 and FT246 cells using FGFR siRNAs or blockage of FGFR activities using BGJ398 eliminate YAP-induced or constitutively active YAP-induced cell growth. Each bar represents mean \pm s.e.m. of four independent repeats. Bars with different letters are significantly different from each other $(P<0.05)$. (b) Verteporfin (YAP antagonist, $5 \mu \mathrm{m}$ ) and BGJ398 (FGFR inhibitor, $1 \mu \mathrm{m}$ ) block YAP-induced growth of FT194 cells in a 3D hanging drop culture system. (c) Representative images from the soft agar assays showing that verteporfin and BGJ398 block YAP-induced colony formation in FT194 cells. (d) Fluorescence-based quantitative soft agar assay showing that verteporfin and BGJ398 block YAP-induced colony formation in FT194 cells. Each bar represents mean \pm s.e.m. of four independent repeats. Bars with different letters are significantly different from each other $(P<0.05)$. (e) Representative images showing that treatment of tumor tissues derived from transformed FT194 cell mouse xenografts with BGJ398 (BGJ) or verteporfin (VTPF) suppressed tumor cell growth, which was indicated by the reduced expression of Ki-67 (Ki67-positive cells are in green) (lower panel), and increased tumor cell apoptosis, which is indicated by TUNEL staining (TUNEL-positive tumor cells are in green) (upper panel). Scale bar: $10 \mu \mathrm{m}$.

Therefore, the pathological relevance of HGSC models using HRAS is questionable. In this study, we found that overexpression of the YAP gene is not only sufficient to transform the FTSECs immortalized by viral genes (FT194 cells, immortalized by SV40 $\mathrm{T} / \mathrm{t}$ and $\mathrm{hTERT}$ ), but also FTSEC cells immortalized without viral genes $^{30}$ (FT246, immortalized by TP53 shRNA, CDK ${ }^{\text {R24C }}$ and PP2AB56y shRNA). Wild-type or mutant YAP-overexpressing cells,

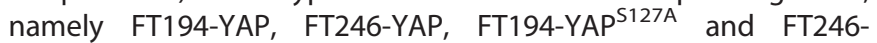
$\mathrm{YAP}^{\mathrm{S127A}}$ cells, overcome contact inhibition and continue to grow after cells reach confluence. Further, these cells acquire the ability of anchorage-independent growth, and form solid tumors in athymic nude mice after implantation. These pieces of evidence clearly position YAP as a critical oncogenic molecule in the development of malignancy in FTSECs. This idea is further supported by the fact that blocking the action of YAP protein using verteporfin, a YAP antagonist, eliminates YAP-induced FTSEC cell proliferation in $3 \mathrm{D}$ culture system and colony formation in the soft agar gel. 
Importantly, we note that tumor cells in FTSEC-derived tumor tissues have pleomorphic nuclei, prominent nucleoli, and are highly proliferative (high mitotic index and Ki-67 expression), suggesting these tumor cells are HGSC cells. As serous tumors usually show positive staining for cytokeratin 7 and negative staining for cytokeratin $20,{ }^{36}$ the strong cytoplasm staining of cytokeratin 7 and negative staining of cytokeratin 20 in FTSEC-derived tumor tissues suggests that these tumors are serous type. Very strong nuclear staining of WT-1 in FTSEC-derived tumor tissues also differentiates them from other types of ovarian tumors, such as endometrioid and clear cell carcinomas. ${ }^{58,59}$ The histological features and the negative periodic acid-Schiff and Alcian blue pH 2.5 staining (Figure $5 \mathrm{~b}$ ) excluded these tumors from the mucinous type. Moreover, compared with HGSC, borderline serous tumors and low-grade serous carcinomas are more likely to show nuclear staining for PAX2. ${ }^{37}$ The very low expression of PAX2 in FTSEC-derived tumor tissues detected using immunohistochemical and western blot analyses indicates that these tumor tissues are not borderline serous tumors or low-grade serous carcinomas. On the other hand, PAX8 is a reliable marker of female genital tract tumors and is almost always positive in serous tumors. ${ }^{31,33,34}$ The very strong nuclear staining of PAX8 further confirms that FTSEC-derived tumors are HGSC in nature. Consistent with our findings, two very recent studies using FTSECs to model the cell origin of ovarian surface epithelial also indicate that tumors derived from FTSEC are HGSC in nature. ${ }^{21,22}$ Although another research group found that transfection of the SV40 T and hTERT immortalized fallopian tube epithelial cell lines with mutated HRAS induced poorly differentiated mucinous adenocarcinoma mixed with undifferentiated carcinoma, the authors believe that this discrepancy could be attributed to the purity of cells used in their study. ${ }^{56}$ Moreover, findings from three very recent transgenic mouse models also strongly suggest that the tumors derived from FTSECs are HGSC. ${ }^{31,60,61}$

Molecular mechanisms underlying the initiation and progression of FTSEC-derived fallopian tube and ovarian HGSCs are unknown. It is known that the mitogen FGFs have key roles in the growth and survival of progenitor cells during development, tissue regeneration and carcinogenesis. ${ }^{62,63}$ Studies on the gene expression profiling of advanced ovarian cancer suggest that FGF2 acts as an autocrine growth factor for ovarian cancer cell proliferation and invasion, ${ }^{38,64-66}$ and tissue healing. ${ }^{67,68}$ Previous research also demonstrates that cultured FTSECs produce FGF2. ${ }^{23}$ Consistent with these results, we show that FTSECs not only secret FGF1 and FGF2, but also express all four FGFRs, suggesting that FGF1/2 may have autocrine and/or paracrine roles in the fallopian tube cells. Supporting our hypothesis, this study clearly shows that FGF1 and FGF2 can stimulate cell proliferation, promote cell cycle progression and enhance migration in FTSECs. These findings strongly indicate that FGFs are functional mitogens in FTSECs. Intriguingly, we found that ectopic expression of YAP or constitutively active YAP not only stimulates expression and secretion of FGF1/2, but also induces the expression of FGFRs. It seems that in the FTSECs, YAP may function as a major factor in the FGF signaling, which has critical roles in regulating growth of FTSECs in an autocrine/paracrine manner. Consistent with these observations, knockdown of YAP eliminated FGF-stimulated proliferation and migration of FTSECs. Moreover, treatment of FTSECs with verteporfin, a YAP antagonist, entirely blocked YAPand $Y A P^{S 127 A}$-induced production of FGF1/2. Finally, we found that FGF2-induced FGF1 and FGF2 mRNA expression in FTSECs (Figure 8d), knockdown of YAP blocked FGF2-induced FGF1/2 mRNA expression (Figure 8d). Clearly, the FGFR signaling pathway formed an autocrine loop to regulate the growth of FTSECs. YAP is required for FGF2 to promote proliferation and migration of normal and transformed FTSECs.

YAP is the major effector of the Hippo pathway, although the Hippo-independent YAP action has also been reported. ${ }^{12,16}$ FGF2 treatment suppressed the Hippo pathway and activated YAP in FTSECs (Figure 9a), suggesting that the Hippo pathway is involved in the interaction between FGF/FGFR and YAP in the FTSECs. Knockdown of LATS1/2 significantly promoted proliferation and migration of FTSECs. Knockdown of LATS1/2 also stimulated expression of FGF ligands and FGFRs (Supplementary Figure 19). Furthermore, both BGJ398 (FGFR antagonist) and verteporfin (YAP antagonist) eliminated LATS1/2 knoockdown-induced proliferation and migration of FTSECs (Figure 9). These lines of evidence clearly indicated that the Hippo/YAP pathway and the FGFR signaling pathway interact with each other to control the growth of FTSECs.

MAPK and PI3K/AKT pathways are two known major downstream pathways of the FGF signaling cascade. ${ }^{42,69}$ Pretreatment of FTSECs with BGJ398 (a pan FGFR inhibitor), LY294002 (a PI3K inhibitor) or U0126 (a MEK inhibitor) blocked FGF2-induced dephosphorylation of YAP, suggesting the PI3K and MAPK pathways are actively involved in FGF2-induced suppression of the Hippo pathway and activation of YAP oncogene. Inhibition of YAP- or YAP ${ }^{\text {127A }}$-induced spheroid growth by U0126 and LY294002, and activation of ERK1/2 and AKT by knockdown of LATS1/2 in FTSECs clearly indicate the involvement of ERK1/2 and AKT pathway in the crosstalk between the Hippo pathway and the FGFR pathway in the FTSECs. Treatment of FTSECs with FGF2 rapidly (within $10 \mathrm{~min}$ ) activates ERK $1 / 2$ and AKT pathway, but suppresses the Hippo signaling pathway 30-60 min after treatment (Figure 9a), suggesting that ERK $1 / 2$ and AKT are potential mediators of the interactions between the Hippo/YAP pathway and the FGFR pathway. Collectively, these results suggest that MAPK and AKT pathways are actively involved in the interaction between the Hippo/YAP pathway and the FGFR pathway. However, more experiments are necessary for us to uncover the exact molecular base underlying the proper crosstalk among these pathways in the FTSECs.

In summary, our study suggests that YAP is overexpressed in the fallopian tube inflammatory and cancerous tissues. Overexpression or constitutive activation of YAP promote proliferation and drive transformation of FTSECs. Moreover, we found that YAP stimulates secretion of FGF ligands and expression of FGFRs. Elevated FGF ligands bind to the increased FGF receptors to activate downstream signaling pathways such as PI3K and MAPK pathways, leading the suppression of the Hippo pathway and activation of YAP protein. Activated YAP can, in turn, stimulate the proliferation of FTSECs and enhance the production of FGFs and expression of FGFRs. Our study clearly suggests that the Hippo/YAP signaling pathway interacts with FGF signaling pathway to form an autocrine/paracrine loop to induce the growth of transformed Fallopian tube epithelial cells (Figure 11a). The existence of the positive feedback loop is confirmed and clinical relevance of this positive regulatory loop is evidenced by the Multidimensional Genomics Data analysis using online cancer database and data-mining tools (TCGA and the cBioPortal for Cancer Genomics). ${ }^{26,27}$ These analyses indicate that $77 \%(322 / 420)$ of ovarian HGSCs have alterations in major components of this FGFs/FGFR/Hippo/YAP/FGFs positive feedback loop (Figure 11b). These alterations match perfectly with our present finding (downregulation of LATS1 and upregulation of YAP, TEAD, FGF1/2, FGFRs and $P I 3 K$ ) (Figure $11 \mathrm{~b}$ ). Several phase I and phase II clinical trials for BGJ398, a pan FGFR inhibitor, are currently underway to examine the role of this novel small molecule in the treatment of several solid tumors (http://clinicaltrials.gov/ct2/results?term = BGJ398\& Search = Search). Evidence in this study clearly suggests that combined targeting of YAP with a YAP inhibitor (such as verteporfin) and FGFRs with a FGFR inhibitor (such as BGJ398) represents a novel therapeutic strategy for the FTSEC-derived fallopian tube and ovarian HGSC. 

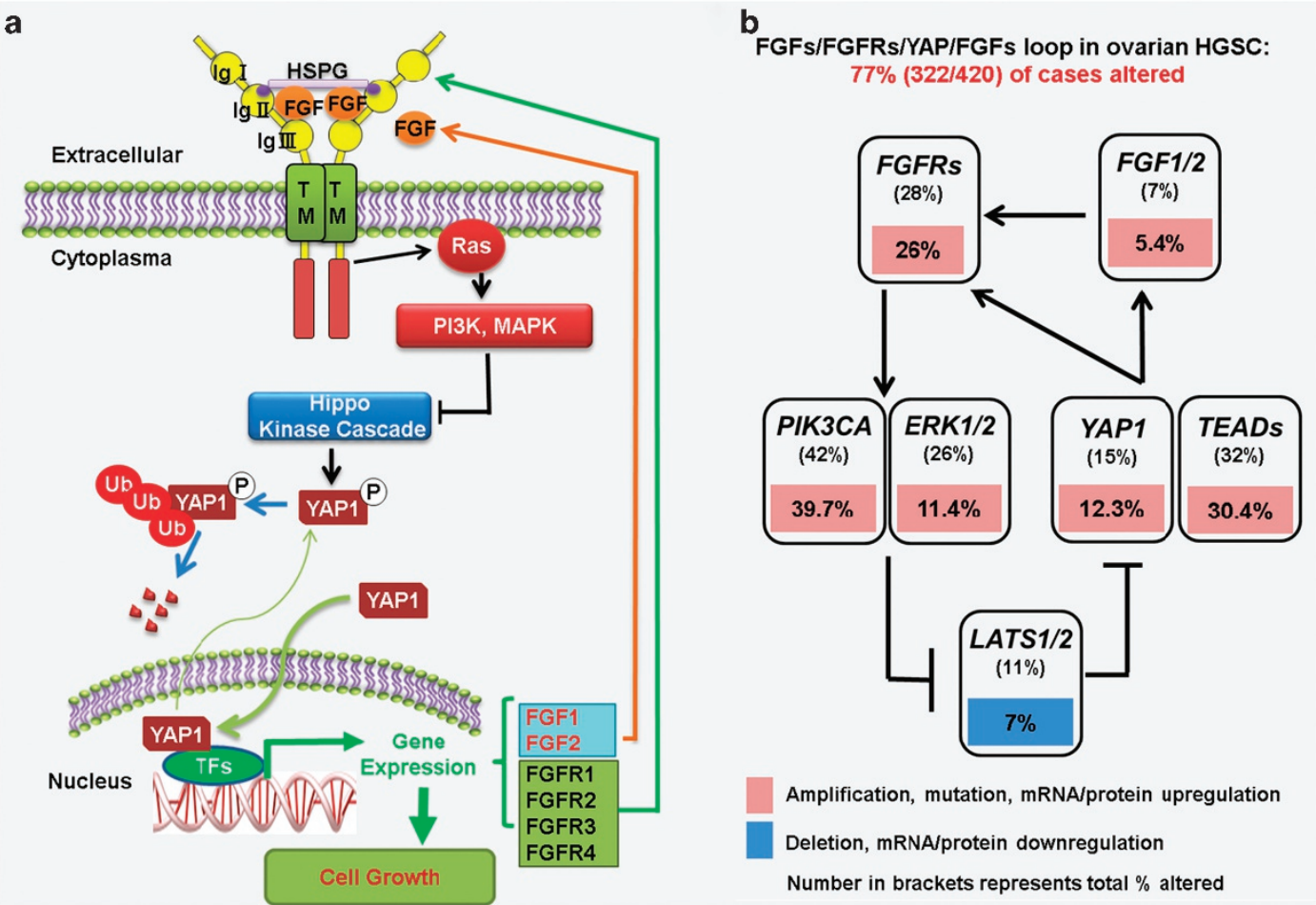

Figure 11. Schematic diagram showing the signaling mechanisms underlying the Hippo/YAP pathway regulation of ovarian HGSC progression. (a) Schematic diagram showing that the Hippo/YAP and FGF/FGFR pathways interact with each other to form a positive feedback loop to regulate activities of FTSECs. Our data indicated that dysfunction of the Hippo signaling pathway may lead to dysregulation of YAP protein expression or activation. Overexpression or constitutive activation of YAP protein in FTSECs is able to promote proliferation and drive transformation of these cells. Moreover, increased YAP activation stimulates secretion of FGF ligands and expression of FGFRs. Elevated FGF ligands bind to the increased FGF receptors to activate downstream signaling pathways such as PI3K and MAPK pathways, leading the suppression of the Hippo pathway and activation of YAP protein. Dysfunction of FGFR pathway may also have the similar effect on the FTSECs. The Hippo/YAP and FGF/FGFR pathways interact with each other to form a positive feedback loop to regulate activities of FTSECs. (b) The clinical relevance of the discovered positive feedback loop is evidenced by the results from the multidimensional cancer genomics data analysis. These large-scale patient sample analyses indicate that $77 \%$ of ovarian HGSC cases (322/420) have alterations in major genes composing the positive feedback loop.

\section{MATERIALS AND METHODS}

Chemicals

Recombinant human acidic FGF (FGF1) and basic FGF (FGF2), as well as the enzyme-linked immunosorbent assay kits for FGF1 and FGF2 measurements, were from R\&D Systems Inc. (Minneapolis, MN, USA). Dulbecco's modified Eagle's medium/F12 cell culture medium was from Fisher Scientific Inc. (Pittsburgh, PA, USA). The Ultroser G serum substitute was from Pall Corporation (Saint-Germain-en-Laye Cedex, France). Fetal bovine serum was from Atlanta Biologicals, Inc. (Lawrenceville, GA, USA). The complementary DNA first-strand synthesis and PCR chemicals, and Alexaconjugated secondary antibodies were from Life Technologies (Grand Island, NY, USA). The RNeasy Mini Kit was from QIAGEN Inc. (Valencia, CA, USA). YAP siRNA was from Dhamarcon/Thermo Scientific (Pittsburgh, PA, USA). Antibodies against YAP, phospho-YAP (ser127), cyclin A2, cyclin D1, cyclin B1 and cytokerintins were from Cell Signaling Technology Inc. (Danvers, MA, USA); PAX8 and WT-1 were from Proteintech Inc. (Chicago, IL, USA). Peroxidase-conjugated secondary antibodies for western blot analysis were from Jackson Immunoresearch Laboratories Inc. (West Grove, PA, USA). The SuperSignal West Femto Chemiluminescent Substrate Kit was from Pierce/Thermo Scientific (Rockford, IL, USA). Optitran Nitrocellular transfer membrane was from Schleicher \& Schuell Bioscience (Dassel, Germany). Antibodies against $\beta$-actin were from Sigma-Aldrich (St Louis, MO, USA). LY294002 and UO126 inhibitors were from Calbiochem (San Diego, CA, USA). The BGJ398 inhibitor was from Medchem Express (Monmouth Junction, NJ, USA). The matrigel basement membrane matrix was from BD Biosciences Inc. (San Jose, CA, USA).

\section{Cell lines and human ovarian tissues}

FTSECs have been successfully isolated from primary human fallopian tube tissue and immortalized by expressing hTERT and perturbing the TP53 and
RB1 tumor-suppressor pathways. ${ }^{30}$ Immortalized human FTSECs, namely FT190, FT194, FT237, FT240 and FT246, were used in these experiments (passage number $<10$ ). OVSAHO cell line was purchased from the Riken Biosource Center (Riken Cell Bank, Tsukuba, Japan) and has been validated by short tandem repeat polymorphism analysis. Human normal and cancerous fallopian tube and ovarian tissue microarray slides were purchased from US Biomax (Rockville, MD, USA). A total of 29 normal, inflammation and cancerous human fallopian tube tissues (including 10 adenocarcinoma tissues, 10 inflammation tissues and 9 normal fallopian tube tissues) and 147 normal and cancerous ovarian tissues (42 normal, 105 ovarian HGSC) were used for IHC analysis. Each sample from an individual patient was arranged in duplicate on the slides.

Immunohistochemistry and the Alcian blue $\mathrm{pH}$ 2.5-periodic acid-Schiff staining

YAP expression in normal, inflammatory and cancerous human fallopian tube tissues was detected using a peroxidase-based immunohistochemistry as previously described. ${ }^{19}$ Sections were scanned using an iSCAN Coreo Slide Scaner (Ventana Medical Systems, Inc., Oro Valley, AZ, USA). The intensity of the positive signal was quantified and recorded using Aperio ImageScope software (Aperio Technologies, Inc., Vista, CA, USA). The annotations and the positive pixel count $V 9$ parameters were set according to the user instructions to analyze YAP immunosignal (Ip $($ Low $)=\operatorname{Isp}($ High $)$ were set to 100 . All other parameters were set as default). The values of total intensity of positive and positivity were record and analyzed. The positivity (that is, the ratio of positive cells per total cell number) of each section was also recorded. The automatic scores from the software were verified by pathologist (Dr Subodh M Lele).

Immunofluorescent histochemistry was used to localize YAP in the established cell lines. Briefly, cells were fixed in freshly prepared $4 \%$ 
paraformaldehyde and stained for YAP using a protocol established in our laboratory. ${ }^{19,70}$ Images were captured using a Zeiss 710 Meta Confocal Laser Scanning Microscope and analyzed using Zeiss Zen 2010 software (Carl Zeiss Microscopy, LLC, Thornwood, NY, USA).

Alcian Blue 2.5 staining was used to determine the production of sulfated and carboxylated acid mucopolysaccharides and sialomucins in the FTSEC-derived tumor xenografts. The colorectal epithelium tissues were used as a positive control. The section was counter-stained with periodic acid-Schiff and hematoxylin.

\section{Cell proliferation analysis}

Six cell lines expressing different levels of YAP protein were established to determine the effect of YAP on FTSECs proliferation. Briefly, FTSECs were cultured to $40 \%$ confluent and then transfected using retrovirus-based YAP overexpression constructs. The efficiency of these vectors has been reported previously. ${ }^{19,49}$ Stable FT194-MXIV, FT194-YAP and FT194$Y P^{S 127 A}$ expressing clones were selected using G418. YAP expression and phosphorylation in these cells were confirmed by western blot. The following six stable cell lines were established: (1) FT194-MXIV, (2) FT246-MXIV, (3) FT194-YAP, (4) FT246-YAP, (5) FT194-YAP $127 A$ and (6) FT246-YAP ${ }^{\text {S127A }}$. The FT194-MXIV and FT246-MXIV cell lines were created by transfecting FT194 and FT246 cells with the empty vector (MXIV). These cells expressed endogenous YAP and were used as control cells. The FT194-YAP and FT246-YAP cell lines, which overexpress wild-type YAP protein, were created by transfecting FT194 and FT246 cells with vectors expressing wild-type YAP. The FT194-YAP S127A and FT246-YAP ${ }^{\text {S127A }}$ cells were created by transfecting FT194 and FT246 cells with vectors expressing constitutively activated YAP (that is, dephosphorylated YAP). The serine to alanine mutation of YAP at residue 127 (YAP ${ }^{\mathrm{S} 127 \mathrm{~A}}$ ) prevented YAP phosphorylation, leading to its nuclear localization and constitutive activation. YAP expression levels were confirmed by western blot, reverse transcriptase-PCR (RT-PCR) and immunofluorescence assay. Cell growth was determined by counting the cell number using an Invitrogen Countess Automated cell counter (Invitrogen Corporation, Carlsbad, CA, USA).

The effect of YAP on FTSEC proliferation was also determined by knockdown of YAP using a RNA interference technique. Briefly, $60 \%$ confluent FT194 and FT246 cells were transfected with siGLO (a cy5-labeled non-target siRNA as control) or YAP siRNAs for $6 \mathrm{~h}$ using Lipofectamine RNAimax (Invitrogen Corporation). Successful knockdown of YAP was also confirmed by RT-PCR and western blot. Cell growth was determined by counting cell number using an Invitrogen Countess Automated cell counter as described above.

\section{Cell cycle analysis}

FTSECs subjected to different treatments (including control cells, YAP overexpression and constitutively activated YAP, and YAP knockdown cells) were trypsinized, fixed and permeabilized with $70 \%$ ethanol overnight at $-20^{\circ} \mathrm{C}$. Cells were then labeled with propidium iodide for $30 \mathrm{~min}$ at $37^{\circ} \mathrm{C}$ and flow cytometry was used to determine the cell cycle distribution of the cells.

\section{Cell migration assays}

A wound-healing assay was used to test the motility of FTSECs control cells, YAP overexpression, constitutively activated cells, YAP knockdown cells and FGF2 incubated cells. ${ }^{71}$ The 'wound' area was measured using computerized Microsuite FIVE imaging software (Olympus America, Inc., Center Valley, PA, USA). We also use an additional Transwell cell migration assay protocol established in our laboratory to confirm the effect of YAP overexpression or constitutive activation on FESEC migration. ${ }^{71}$ Experiments were repeated in triplicate, and at least three inserts were used for each treatment group.

\section{Colony formation assay}

The effect of YAP on the transformation of non-oncogenic normal FTSECs was determined using a Cytoselect 96-Well Cell Transformation kit (Cell Biolabs, Inc.) according to the manufacturer's instructions. This soft agar-based cell colony formation kit was also used to determine the effect of YAP on the anchorage-independent growth of FTSECs in vitro.
Western blot analysis

Protein levels in FTSECs, as well as in the xenograft tissues, were determined by western blot based on a protocol established in our laboratory. ${ }^{19,72}$ The immunosignal was detected using a Thermo Scientific SuperSignalWest Femto Chemiluminescent Substrate Kit (Thermo Fisher Scientific, Rockford, IL, USA). The images were captured and analyzed with a UVP gel documentation system (UVP, LLC, Upland, CA, USA).

\section{RT-PCR analysis of gene expression}

Expression of YAP, AREG, FGF ligands and FGFRs were also detected with RT-PCR. Total RNA was prepared by TRIzol reagent (Invitrogen Corporation), and reverse transcription was completed using a SuperScript III First-Strand Synthesis System (Life TechnlogyTM, Grand Island, NY, USA). RT-PCR was performed on an MJ Research PTC100 Programmable Thermal cycler (Bio-Rad Laboratories, Inc., Hercules, CA, USA) based on a protocol established in our laboratory. ${ }^{19,71}$ Primers have been described and validated previously. ${ }^{73,74}$

\section{In vivo tumorigenicity}

To confirm the transformative action of YAP in the FTSECs, FT194-MXIV, FT194-YAP and FT194-YAP ${ }^{5127 A}$ cells were suspended in $100 \mu \mathrm{l}$ of Dulbecco's modified Eagle's medium/F12, mixed with $100 \mu \mathrm{l}$ of Matrigel (BD Biosciences Inc.) and injected subcutaneously into the left shoulder (MXIV control) and right shoulder (YAP overexpression or constitutively activated) of 5-week-old female athymic nude mice $(n=6)$. The tumor diameter was recorded weekly for 11 weeks. Tumor volume $\left(\mathrm{mm}^{3}\right)$ was estimated by measuring the longest and shortest diameter of the tumor and calculated as follows: volume $=(\text { shortest diameter })^{2} \times$ (longest diameter) $\times 3.14 \div 6$. All mice were killed at the end of the experiment, and tumors were collected for preparation of protein, RNA and paraffin tissue sections. The animal handling procedures and all experimental protocols were approved by the Institutional Animal Care and Use Committee (IACUC) at the University of Nebraska Medical Center.

\section{Multidimensional oncogenomic data analysis}

Multidimensional cancer genomic data analysis was performed using online data-mining tool from the cBioPortal for Cancer Genomics (http:// cbioportal.org) and the data sets from the cBioPortal for Cancer Genomics and the TCGA research Network (http://cancergenome.nih.gov) according to recently published protocols. ${ }^{26,27}$ Tumor types and data sets are chosen in accordance with the publication guidelines from TCGA (last update: 30 September 2014). Genomic alterations are identified when the following occurs: (1) gene mutations; (2) putative copy number alteration (amplification or deletion); (3) RNA expression Z-scores (RNA Seq Version 2 RSEM) with Z-score thresholds \pm 2.0 ; and (4) protein/phospho-protein level (RPPA) with Z-score thresholds \pm 2.0 . All clinical data and copy number alterations or variations data were merged per 'Case Id' as the linking variable after the separate files were uploaded to (GraphPad Software, Inc., La Jolla, CA, USA) for statistical analysis.

Xenograft tumor tissue culture

FT194-YAP S127A cells (transformed FT194 cells) were injected subcutaneously into the athymic nude mice to form HGSC tumors as described in Figure 4. When tumor size (diameter) reached $0.8 \mathrm{~cm}$, the nude mice were killed and the tumors were surgically removed, sliced into $300 \mu \mathrm{m}$ slices. The tumor slice was then cut into small pieces $(1.0 \mathrm{~mm} \times 1.0 \mathrm{~mm})$ under a stereo microscope and loaded onto the membrane of a culture insert in a tissues culture system, which has been reported previously. ${ }^{70}$ Tumor tissues were culture for 3 days with or without BGJ $(1 \mu \mathrm{m})$ or verteporfin $(5 \mu \mathrm{m})$. Control tissues were treated with the same amount of vehicle (dimethylsulfoxide, $0.1 \%$ final concentration). The incubated tissues were embedded into optimal cutting temperature compound and sectioned using a cryostat to prepare the frozen section for TUNEL assay and Ki-67 staining. TUNEL assay and $\mathrm{Ki}-67$ staining were performed with protocols established in our laboratory. ${ }^{20}$ OVSAHO, a verified high-grade ovarian serous carcinoma cell lines, ${ }^{43,75}$ have been used to replace the FT194YAP $^{\text {S127A }}$ cells to confirm the role of verteporfin and BGJ398 on the growth of ovarian HGSC tumor in vitro using the same experimental design. 


\section{Statistical analysis}

All experiments were repeated at least three times with each sample in triplicate unless otherwise noted (duplicate only if the volume of a sample is limited). Statistical analyses were conducted primarily using GraphPad Prism software (GraphPad Software, Inc.). Statistical comparisons between two groups were analyzed for significance by one-way analysis of variance with Tukey's post-Hoc test or by two-tailed Student's $t$-test. Multiple group comparisons were assessed using two-way repeated-measures analysis of variance with post-hoc $t$-test. Data in the graph are presented as the mean \pm s.e.m. Sample numbers used for cell biology and biochemical studies were determine based on data reported previously. ${ }^{20}$ For tumorigenic experiments, six animals were used in each groups. The number was determined by power analysis based on a preliminary study and a relevant report. ${ }^{20}$ Survival data were estimated by Kaplan-Meier method analyzed by log-rank test. HGSC YAP expression data and patient survival data were extracted from TCGA database (http://cancergenome. nih.gov/). The frequency of cross-cancer YAP gene alterations and the frequency of YAP/TEADs gene alteration in ovarian HGSC were analyzed and graphed by the cBioPortal online analysis tool (the cBioportal for Cancer Genomics, http://www.cbioportal.org/) using data extracted from TCGA. 26,27

\section{CONFLICT OF INTEREST}

The authors declare no conflict of interest.

\section{ACKNOWLEDGEMENTS}

We thank Dr Adam Karpf (The Pamela and Fred Cancer Center, University of Nebraska Medical Center) for providing ovarian HGSC cell lines. We acknowledge the cBioPortal for Cancer Genomics (http://cbioportal.org) for providing data sets and online analyzing tools. We also acknowledge the TCGA research Network (http:// cancergenome.nih.gov) for providing data sets. This work was supported by the Eunice Kennedy Shriver National Institute of Child Health and Human Development (5R00HD059985, 5P01AG029531); the Olson Center for Women's Health (no number); the Fred and Pamela Buffett Cancer Center (LB595), the Colleen's Dream Foundation (no number), the Omaha Veterans Administration Medical Center (5101BX000512) and the National Institute of Food and Agriculture (2011-67015-20076). We thank Melody Montgomery at the University of Nebraska Medical Center (UNMC) Research Editorial Office for the professional editing of the manuscript.

\section{REFERENCES}

1 Jemal A, Bray F, Center MM, Ferlay J, Ward E, Forman D. Global cancer statistics. CA Cancer J Clin 2011; 61: 69-90.

2 Seidman JD, Horkayne-Szakaly I, Haiba M, Boice CR, Kurman RJ, Ronnett BM. The histologic type and stage distribution of ovarian carcinomas of surface epithelial origin. Int J Gynecol Pathol 2004; 23: 41-44.

3 Selvaggi SM. Tumors of the ovary, maldeveloped gonads, fallopian tube, and broad ligament. Arch Pathol Lab Med 2000; 124: 477.

4 Auersperg N, Wong AS, Choi KC, Kang SK, Leung PC. Ovarian surface epithelium: biology, endocrinology, and pathology. Endocr Rev 2001; 22: 255-288.

5 Katabuchi $\mathrm{H}$, Okamura $\mathrm{H}$. Cell biology of human ovarian surface epithelial cells and ovarian carcinogenesis. Med Electron Microsc 2003; 36: 74-86.

6 Casey MJ, Bewtra C, Hoehne LL, Tatpati AD, Lynch HT, Watson P. Histology of prophylactically removed ovaries from BRCA1 and BRCA2 mutation carriers compared with noncarriers in hereditary breast ovarian cancer syndrome kindreds. Gynecol Oncol 2000; 78: 278-287.

7 Barakat RR, Federici MG, Saigo PE, Robson ME, Offit K, Boyd J. Absence of premalignant histologic, molecular, or cell biologic alterations in prophylactic oophorectomy specimens from BRCA1 heterozygotes. Cancer 2000; 89: 383-390.

8 Berek JS, Crum C, Friedlander M. Cancer of the ovary, fallopian tube, and peritoneum. Int J Gynaecol Obstet 2012; 119: S118-S129.

9 Kurman RJ, Shih leM. Molecular pathogenesis and extraovarian origin of epithelial ovarian cancer--shifting the paradigm. Hum Pathol 2011; 42: 918-931.

10 Kindelberger DW, Lee Y, Miron A, Hirsch MS, Feltmate C, Medeiros F et al. Intraepithelial carcinoma of the fimbria and pelvic serous carcinoma: evidence for a causal relationship. Am J Surg Pathol 2007; 31: 161-169.

11 Goodman MT, Shvetsov YB. Incidence of ovarian, peritoneal, and fallopian tube carcinomas in the United States, 1995-2004. Cancer Epidemiol Biomarkers Prev 2009; 18: 132-139.

12 Johnson R, Halder G. The two faces of Hippo: targeting the Hippo pathway for regenerative medicine and cancer treatment. Nat Rev Drug Discov 2014; 13: 63-79.
13 Pan D. The Hippo signaling pathway in development and cancer. Dev Cell 2010; 19: 491-505.

14 Huang J, Wu S, Barrera J, Matthews K, Pan D. The Hippo signaling pathway coordinately regulates cell proliferation and apoptosis by inactivating Yorkie, the Drosophila homolog of YAP. Cell 2005; 122: 421-434.

15 Tapon N, Harvey KF, Bell DW, Wahrer DC, Schiripo TA, Haber D et al. Salvador promotes both cell cycle exit and apoptosis in Drosophila and is mutated in human cancer cell lines. Cell 2002; 110: 467-478.

16 Yu FX, Guan KL. The Hippo pathway: regulators and regulations. Genes Dev 2013; 27: $355-371$

17 Zhang X, George J, Deb S, Degoutin JL, Takano EA, Fox SB et al. The Hippo pathway transcriptional co-activator, YAP, is an ovarian cancer oncogene. Oncogene 2011; 30: 2810-2822.

18 Hall CA, Wang R, Miao J, Oliva E, Shen X, Wheeler T et al. Hippo pathway effector Yap is an ovarian cancer oncogene. Cancer Res 2010; 70: 8517-8525.

19 Fu D, Lv X, Hua G, He C, Dong J, Lele SM et al. YAP regulates cell proliferation, migration, and steroidogenesis in adult granulosa cell tumors. Endocr Relat Cancer 2014; 21: 297-310.

20 He C, Lv X, Hua G, Lele SM, Remmenga S, Dong J et al. YAP forms autocrine loops with the ERBB pathway to regulate ovarian cancer initiation and progression. Oncogene 2015; e-pub ahead of print 23 March 2015; doi:10.1038/ onc.2015.52.

21 Jazaeri AA, Bryant JL, Park H, Li H, Dahiya N, Stoler MH et al. Molecular requirements for transformation of fallopian tube epithelial cells into serous carcinoma. Neoplasia 2011; 13: 899-911.

22 Karst AM, Levanon K, Drapkin R. Modeling high-grade serous ovarian carcinogenesis from the fallopian tube. Proc Natl Acad Sci USA 2011; 108: 7547-7552.

23 Ando H, Kobayashi M, Toda S, Kikkawa F, Masahashi T, Mizutani S. Establishment of a ciliated epithelial cell line from human Fallopian tube. Hum Reprod 2000; 15: 1597-1603.

24 Smith JA, Madden T, Vijjeswarapu M, Newman RA. Inhibition of export of fibroblast growth factor-2 (FGF-2) from the prostate cancer cell lines PC3 and DU145 by Anvirzel and its cardiac glycoside component, oleandrin. Biochem Pharmacol 2001; 62: 469-472.

25 Guagnano V, Furet P, Spanka C, Bordas V, Le Douget M, Stamm C et al. Discovery of 3-(2,6-dichloro-3,5-dimethoxy-phenyl)-1-\{6-[4-(4-ethyl-piperazin-1yl)-phenylamin o]-pyrimidin-4-yl\}-1-methyl-urea (NVP-BGJ398), a potent and selective inhibitor of the fibroblast growth factor receptor family of receptor tyrosine kinase. J Med Chem 2011; 54: 7066-7083.

26 Gao J, Aksoy BA, Dogrusoz U, Dresdner G, Gross B, Sumer SO et al. Integrative analysis of complex cancer genomics and clinical profiles using the cBioPortal. Sci Signal 2013; 6: pl1.

27 Cerami E, Gao J, Dogrusoz U, Gross BE, Sumer SO, Aksoy BA et al. The cBio cancer genomics portal: an open platform for exploring multidimensional cancer genomics data. Cancer Discov 2012; 2: 401-404.

28 Cancer Genome Atlas N, Comprehensive molecular portraits of human breast tumours. Nature 2012; 490: 61-70.

29 Cancer Genome Atlas Research N, Kandoth C, Schultz N, Cherniack AD, Akbani R, Liu $Y$ et al. Integrated genomic characterization of endometrial carcinoma. Nature 2013; 497: 67-73.

30 Karst AM, Drapkin R. Primary culture and immortalization of human fallopian tube secretory epithelial cells. Nat Protoc 2012; 7: 1755-1764.

31 Perets R, Wyant GA, Muto KW, Bijron JG, Poole BB, Chin KT et al. Transformation of the fallopian tube secretory epithelium leads to high-grade serous ovarian cancer in Brca;Tp53;Pten models. Cancer Cell 2013; 24: 751-765.

32 Kuhn E, Meeker A, Wang TL, Sehdev AS, Kurman RJ, Shih leM. Shortened telomeres in serous tubal intraepithelial carcinoma: an early event in ovarian high-grade serous carcinogenesis. Am J Surg Pathol 2010; 34: 829-836.

33 Bowen NJ, Logani S, Dickerson EB, Kapa LB, Akhtar M, Benigno BB et al. Emerging roles for PAX8 in ovarian cancer and endosalpingeal development. Gynecol Oncol 2007; 104: 331-337.

34 Laury AR, Hornick JL, Perets R, Krane JF, Corson J, Drapkin R et al. PAX8 reliably distinguishes ovarian serous tumors from malignant mesothelioma. Am J Surg Pathol 2010; 34: 627-635.

35 Madore J, Ren F, Filali-Mouhim A, Sanchez L, Kobel M, Tonin PN et al. Characterization of the molecular differences between ovarian endometrioid carcinoma and ovarian serous carcinoma. J Pathol 2010; 220: 392-400.

36 Cathro HP, Stoler MH. Expression of cytokeratins 7 and 20 in ovarian neoplasia. Am J Clin Pathol 2002; 117: 944-951.

37 Tung CS, Mok SC, Tsang YT, Zu Z, Song H, Liu J et al. PAX2 expression in low malignant potential ovarian tumors and low-grade ovarian serous carcinomas. Mod Pathol 2009; 22: 1243-1250.

38 Crickard K, Gross JL, Crickard U, Yoonessi M, Lele S, Herblin WF et al. Basic fibroblast growth factor and receptor expression in human ovarian cancer. Gynecol Oncol 1994; 55: 277-284. 
39 Liu-Chittenden Y, Huang B, Shim JS, Chen Q, Lee SJ, Anders RA et al. Genetic and pharmacological disruption of the TEAD-YAP complex suppresses the oncogenic activity of YAP. Genes Dev 2012; 26: 1300-1305.

40 Zhang J, Ji JY, Yu M, Overholtzer M, Smolen GA, Wang R et al. YAP-dependent induction of amphiregulin identifies a non-cell-autonomous component of the Hippo pathway. Nat Cell Biol 2009; 11: 1444-1450.

41 Yang S, Zhang L, Liu M, Chong R, Ding SJ, Chen Y et al. CDK1 phosphorylation of YAP promotes mitotic defects and cell motility and is essential for neoplastic transformation. Cancer Res 2013; 73: 6722-6733.

42 Fearon AE, Gould CR, Grose RP. FGFR signalling in women's cancers. Int J Biochem Cell Biol 2013; 45: 2832-2842.

43 Domcke S, Sinha R, Levine DA, Sander C, Schultz N. Evaluating cell lines as tumour models by comparison of genomic profiles. Nat Commun 2013; 4: 2126.

44 Friedrich J, Seidel C, Ebner R, Kunz-Schughart LA. Spheroid-based drug screen: considerations and practical approach. Nat Protoc 2009; 4: 309-324.

45 Stewart SL, Wike JM, Foster SL, Michaud F. The incidence of primary fallopian tube cancer in the United States. Gynecol Oncol 2007; 107: 392-397.

46 Crum CP, Drapkin R, Miron A, Ince TA, Muto M, Kindelberger DW et al. The distal fallopian tube: a new model for pelvic serous carcinogenesis. Curr Opin Obstet Gynecol 2007; 19: 3-9.

47 Dubeau L, Drapkin R. Coming into focus: the nonovarian origins of ovarian cancer. Ann Oncol 2013; 24: viii28-viii35.

48 Levanon K, Crum C, Drapkin R. New insights into the pathogenesis of serous ovarian cancer and its clinical impact. J Clin Oncol 2008; 26: 5284-5293.

49 Dong J, Feldmann G, Huang J, Wu S, Zhang N, Comerford SA et al. Elucidation of a universal size-control mechanism in Drosophila and mammals. Cell 2007; 130: 1120-1133.

50 Lee KP, Lee JH, Kim TS, Kim TH, Park HD, Byun JS et al. The Hippo-Salvador pathway restrains hepatic oval cell proliferation, liver size, and liver tumorigenesis. Proc Natl Acad Sci USA 2010; 107: 8248-8253.

51 Harvey KF, Zhang X, Thomas DM. The Hippo pathway and human cancer. Nat Rev Cancer 2013; 13: 246-257.

$52 \mathrm{Cai} \mathrm{H}, \mathrm{Xu}$ Y. The role of LPA and YAP signaling in long-term migration of human ovarian cancer cells. Cell Commun Signal 2013; 11: 31.

53 Yuan M, Tomlinson V, Lara R, Holliday D, Chelala C, Harada T et al. Yes-associated protein (YAP) functions as a tumor suppressor in breast. Cell Death Differ 2008; 15: 1752-1759.

54 Strano S, Monti O, Pediconi N, Baccarini A, Fontemaggi G, Lapi E et al. The transcriptional coactivator Yes-associated protein drives p73 gene-target specificity in response to DNA Damage. Mol Cell 2005; 18: 447-459.

55 Kendall SD, Linardic CM, Adam SJ, Counter CM. A network of genetic events sufficient to convert normal human cells to a tumorigenic state. Cancer Res 2005 . 65: 9824-9828.

56 Shan W, Mercado-Uribe I, Zhang J, Rosen D, Zhang S, Wei J et al. Mucinous adenocarcinoma developed from human fallopian tube epithelial cells through defined genetic modifications. Cell Cycle 2012; 11: 2107-2113.

57 Cancer Genome Atlas Research N, Integrated genomic analyses of ovarian carcinoma. Nature 2011; 474: 609-615.

58 Hwang $\mathrm{H}$, Quenneville L, Yaziji H, Gown AM. Wilms tumor gene product: sensitive and contextually specific marker of serous carcinomas of ovarian surface epithelial origin. Appl Immunohistochem Mol Morphol 2004; 12: 122-126.
59 Al-Hussaini M, Stockman A, Foster H, McCluggage WG. WT-1 assists in distinguishing ovarian from uterine serous carcinoma and in distinguishing between serous and endometrioid ovarian carcinoma. Histopathology 2004; 44: 109-115.

60 Kim J, Coffey DM, Creighton CJ, Yu Z, Hawkins SM, Matzuk MM. High-grade serous ovarian cancer arises from fallopian tube in a mouse model. Proc Natl Acad Sci USA 2012; 109: 3921-3926.

61 Sherman-Baust CA, Kuhn E, Valle BL, Shih leM, Kurman RJ, Wang TL et al. A genetically engineered ovarian cancer mouse model based on fallopian tube transformation mimics human high-grade serous carcinoma development. J Pathol 2014; 233: 228-237.

62 Dailey L, Ambrosetti D, Mansukhani A, Basilico C. Mechanisms underlying differential responses to FGF signaling. Cytokine Growth Factor Rev 2005; 16: 233-247.

63 Eswarakumar VP, Lax I, Schlessinger J. Cellular signaling by fibroblast growth factor receptors. Cytokine Growth Factor Rev 2005; 16: 139-149.

64 De Cecco L, Marchionni L, Gariboldi M, Reid JF, Lagonigro MS, Caramuta S et al. Gene expression profiling of advanced ovarian cancer: characterization of a molecular signature involving fibroblast growth factor 2. Oncogene 2004; 23: 8171-8183.

65 Di Blasio AM, Cremonesi L, Vigano P, Ferrari M, Gospodarowicz D, Vignali M et al. Basic fibroblast growth factor and its receptor messenger ribonucleic acids are expressed in human ovarian epithelial neoplasms. Am J Obstet Gynecol 1993; 169: 1517-1523.

$66 \mathrm{Li} \mathrm{T}$, Jiang S. Effect of bFGF on invasion of ovarian cancer cells through the regulation of Ets-1 and urokinase-type plasminogen activator. Pharm Biol 2010; 48: $161-165$.

67 Meyer GE, Yu E, Siegal JA, Petteway JC, Blumenstein BA, Brawer MK. Serum basic fibroblast growth factor in men with and without prostate carcinoma. Cancer 1995; 76: 2304-2311.

68 Ono I. The effects of basic fibroblast growth factor (bFGF) on the breaking strength of acute incisional wounds. J Dermatol Sci 2002; 29: 104-113.

69 Beenken A, Mohammadi M. The FGF family: biology, pathophysiology and therapy. Nat Rev Drug Discov 2009; 8: 235-253.

70 Wang C, Roy SK. Expression of E-cadherin and N-cadherin in perinatal hamster ovary: possible involvement in primordial follicle formation and regulation by follicle-stimulating hormone. Endocrinology 2010; 151: 2319-2330.

71 Wang C, Lv X, He C, Hua G, Tsai MY, Davis JS. The G-protein-coupled estrogen receptor agonist G-1 suppresses proliferation of ovarian cancer cells by blocking tubulin polymerization. Cell Death Dis 2013; 4: e869.

72 Wang C, Lv X, Jiang C, Cordes CM, Fu L, Lele SM et al. Transforming growth factor alpha (TGFalpha) regulates granulosa cell tumor (GCT) cell proliferation and migration through activation of multiple pathways. PLoS One 2012; 7: e48299.

73 Siffroi-Fernandez S, Cinaroglu A, Fuhrmann-Panfalone V, Normand G, Bugra K, Sahel J et al. Acidic fibroblast growth factor (FGF-1) and FGF receptor 1 signaling in human Y79 retinoblastoma. Arch Ophthalmol 2005; 123: 368-376.

74 Krejci P, Krakow D, Mekikian PB, Wilcox WR. Fibroblast growth factors 1, 2, 17, and 19 are the predominant FGF ligands expressed in human fetal growth plate cartilage. Pediatr Res 2007; 61: 267-272.

75 Beaufort CM, Helmijr JC, Piskorz AM, Hoogstraat M, Ruigrok-Ritstier K, Besselink N et al. Ovarian cancer cell line panel (OCCP): clinical importance of in vitro morphological subtypes. PLoS One 2014; 9: e103988.

Supplementary Information accompanies this paper on the Oncogene website (http://www.nature.com/onc) 